\title{
Forced degradation study of efonidipine HCl ethanolate, characterization of degradation products by LC-Q-TOF-MS and NMR
}

\author{
Charu P. Pandya, Sadhana J. Rajput*
}

Department of Pharmaceutical Quality Assurance, Faculty of Pharmacy, The Maharaja Sayajirao University of Baroda, Center of Relevance and Excellence in New Drug Delivery System, Government of India, Vadodara, Gujarat, India.

\begin{tabular}{l}
\hline ARTICLE INFO \\
\hline Received on: 23/09/2019 \\
Accepted on: 24/12/2019 \\
Available online: 04/04/2020 \\
\hline Key words: \\
Forced degradation, \\
efonidipine, preparative \\
HPLC, LC-Q-TOF-MS, NMR \\
and IR.
\end{tabular}

\begin{abstract}
Efonidipine $\mathrm{HCl}$ Ethanolate is an antihypertensive drug with 1,4 dihydropyridine and phosphinane derivative. Forced degradation study was performed in Efonidipine as per the guidelines by International Conference on Harmonization (ICH) Q1A (R2). Extensive degradation and slight degradation were observed in alkaline and photolytic conditions, respectively, whereas acidic, oxidative, and thermal conditions did not show any degradation. Degradation products were separated on Thermo Hypersil BDS C18 column $(250 \times 4.6 \mathrm{~mm}, 5 \mu)$, mobile phase in gradient mode using ammonium acetate buffer and acetonitrile with detection at a wavelength of $254 \mathrm{~nm}$. Six degradation products in alkaline condition and four degradation products in photolytic condition were identified by HPLC and characterized by mass spectrometry using LC-Q-TOF-MS, and degradation pathway was proposed. This is the typical case of degradation, where co-solvent methanol reacts with Efonidipine to form pseudo degradation products such as DP1, DP4, DP5, and DP6. Three degradation products DP1, DP3, and DP4 in alkaline condition were isolated by preparative HPLC and were characterized by LC-Q-TOF-MS, ${ }^{1} \mathrm{H} /{ }^{13} \mathrm{C}$ NMR, and IR techniques. By characterization with these techniques, DP1 is characterized as 3-2-(N-benzylanilino)ethyl 3-oxo-2,2-dimethylpropyl hydrogen 1,4-dihydro-2,6-dimethyl-4(3-nitrophenyl) pyridin-3-yl-3-phosphonate, DP3 is characterized as 2-(N-benzyl-N-phenylamino) ethanol, and DP4 is characterized as 3-methoxy-2,2-dimethylpropyl hydrogen 1,4-dihydro-2,6-dimethyl-5-methyloxycarbonyl-4-(3nitro)phenylpyridin-3-yl-3-phosphonate. The developed method was validated as per guidelines by ICH with respect to linearity, accuracy, precision, limit of detection, and robustness.
\end{abstract}

\section{INTRODUCTION}

Efonidipine HCL Ethanolate (EFO) is a new calcium channel blocker with dihydropyridine and phosphinane derivative. It blocks both T-type and L-type calcium channels (Hikaru and Koki, 2002; Masuda and Tanaka, 1994; Nakano et al., 2010). It has a slow onset and longer duration of action. In a patient with essential hypertension, it causes an increase in renal blood flow, a

${ }^{*}$ Corresponding Author

Sadhana J. Rajput, Department of Pharmaceutical Quality Assurance, Faculty of Pharmacy, The Maharaja Sayajirao University of Baroda, Center of Relevance and Excellence in New Drug Delivery System, Government of India, Vadodara, Gujarat, India.

E-mail: sjrajput@gmail.com decrease in renal vascular resistance, and an increase in glomerular filtration rate. It chemically consists of 2-(N-benzylanilino) ethyl 5-(5, 5-dimethyl-2-oxo-1, $3, \quad 2 \wedge 5\}$-dioxaphosphinan-2-yl)-2, 6-dimethyl-4-(3-nitrophenyl)-1, 4-dihydropyridine-3-carboxylate, ethanol, hydrochloride with molecular formula $\mathrm{C}_{36} \mathrm{H}_{45} \mathrm{ClN}_{3} \mathrm{O}_{8} \mathrm{P}$, and molecular weight $714.19 \mathrm{~g} / \mathrm{mole}$ (Pubchem, 2019). Efonidipine has pKa (basic) of 2.33 and $\log \mathrm{P}$ is 5.35 (Drugbank, 2019). It was approved in 1995 as a brand name Landel ${ }^{\circledR}$. It is approved for marketing in India by Drug controller general India to Zuventus Pharma as Efnocar ${ }^{\circledR}$. The HPLC method development of EFO has been reported (Kumar et al., 2017). The LC-MS/MS method has been reported for the development of EFO in human plasma for pharmacokinetic applications and its stereospecific determination (Liu et al., 2015; 2016). Literature has been reported on spectroscopic studies on the interaction of efonidipine with bovine serum albumin (Wang et al., 2008), and the development considerations 
for ethanolate with respect to stability and physicochemical considerations of EFO have been reported (Otsuka et al., 2015). Solid dispersions of Efonidipine hydrochloride ethanolate with improved physicochemical and pharmacokinetic properties using microwave treatment have been reported (Otsuka et al., 2016).

Chemical stability of the molecule is an important aspect since it affects the safety and efficacy of the product. There is a requirement of stability testing data from FDA and International Conference on Harmonization (ICH) guidance on how the quality of drug substance and drug product changes with time under the influence of environmental factors. In steps of controlling impurities or degradation products, their identification and characterization are the two main steps. These are performed when impurities or degradation products are present at the prescribed limits of $0.1 \%$ or even lower which are genotoxic in nature. The conventional approach comprises separation of degradation products by a suitable method and identification with the help of standard material. Alternative methods include enrichment or isolation and characterization through spectral techniques (Singh et al., 2012). Sometimes it may not be possible to isolate the degradation products due to their low levels. Presently liquid chromatography/mass spectrometry and NMR techniques are the prominent techniques in structural identification and characterization of degradation products, which shows an important effect on evaluation safety aspects of drugs. The forced degradation helps in the possibility of generation of degradation products, thereby helps in determination of intrinsic stability of molecule and possibility of predicting degradation pathways and validation of stability indicating method (ICH Q1A (R2), 2003).

To the best of our knowledge, there are no reports on the forced degradation study on Efonidipine, identification, isolation, and characterization of degradation products. Our objective is to conduct the forced degradation study of Efonidipine to separate degradation products from Efonidipine, to identify and characterize the degradation products and isolate major degradation products by Preparative HPLC and its characterization using ${ }^{1} \mathrm{H},{ }^{13} \mathrm{C}$ NMR and IR techniques, and finally validation of the developed method as per guidelines by ICH.

\section{EXPERIMENTAL}

\section{Materials and reagents}

EFO was purchased from Shouguang Qihang International Trade Co., China. EFNOCAR ${ }^{\circledR}$ tablet was purchased from the local pharmacy in India. Chemicals used in the analysis were Acetonitrile (HPLC grade), methanol (HPLC grade), and acetic acid (HPLC grade) and were purchased from Rankem Pvt. Ltd, Mumbai, India. Ammonium acetate (HPLC grade), sodium hydroxide, hydrochloric acid, and hydrogen peroxide were purchased from S.D. Fine Chemical Ltd, Mumbai, India.

\section{Equipment and Chromatographic conditions}

HPLC PDA analysis was carried out by Shimadzu (Shimadzu Corporation, Kyoto Japan) containing Shimadzu LC20 AD pump (binary), Shimadzu PDA M-20A diode array detector, and Rheodyne 7725 injector valve with a fixed loop of $20 \mu \mathrm{l}$. LC solution software (Shimadzu Corporation, Japan) was used for data integration. Separation was carried out on Thermo Hypersil BDS C 18 Column $(250 \times 4.6$ mm i.d., $5 \mu$ particle size $)$. Analytes were detected at $254 \mathrm{~nm}$. Buffer used in the mobile phase was acetate buffer $(10 \mathrm{~mm})$, which was prepared by dissolving $770 \mathrm{mg}$ of ammonium acetate in 1,000 $\mathrm{ml}$ of double-distilled water. The $\mathrm{pH}$ was adjusted to 5.8 with acetic acid. Before use, acetate buffer was filtered through $0.2 \mu$ Nylon 6, 6 membrane filter. Mobile

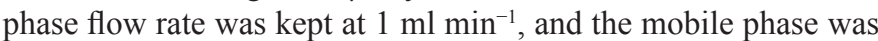
run in gradient program as (time/\% Acetonitrile) $0 / 25,6 / 25,11 / 40$, 35/50, 45/50, 50/53, and 60/53.

Preparative HPLC chromatographic separation was performed on Shimadzu (Shimadzu Corporation, Kyoto, Japan) chromatographic system equipped with the Shimadzu LC-20AP pump (binary) and Shimadzu SPD-20A UV-visible detector. Samples were injected through the Rheodyne 7725 injector valve. Data acquisition and integration were performed using Class VP software. Phenomenex Luna column C $18(250 \times 50 \mathrm{~mm}, 10 \mu)$ was used for isolation of degradation. The flow rate was kept at $50 \mathrm{ml} \mathrm{min}{ }^{-1}$. Detection was performed at $254 \mathrm{~nm}$. The gradient program was (time $/ \%$ Acetonitrile) 0/5, 90/40, and 120/80.

LC-Q-TOF-MS system (Agilent Technologies, Inc, United States) comprising 1290 Infinity UHPLC system, 1260 infinity Nano HPLC with Chipcube, and 6550 ifunnel Q-TOF. Chemstation-LC control software was used for mass spectroscopic studies.

NMR spectroscopy was performed using Bruker Avance II $400 \mathrm{MHz}$ NMR spectrometer for characterization of ${ }^{1} \mathrm{H}$ and ${ }^{13} \mathrm{C}$-NMR spectra having dual broadband probe and $z$-axis gradients. Solvent used for NMR spectra was DMSO- $\mathrm{d}_{6}$, and the internal standard used was tetramethyl silane. Attached Proton test in ${ }^{13} \mathrm{C}$ NMR was conducted to confirm the presence of methyl and methine groups as positive peaks and methylene and quaternary carbon as negative peaks.

IR spectra were recorded on an 8400s spectrophotometer.

Precision water baths (Thermal Lining Services, Vadodara, India) with a temperature controller were used for degradation studies.

Photodegradation studies were performed in a photostability chamber (Thermolab Scientific Equipments Pvt. Ltd., Vadodara, India) with a light bank containing four UV (Osram L73) and fluorescent (L20) lamps (ICH Q1B, 1996).

\section{PREPARATION OF EFO STOCK SOLUTION}

Stock solution of $1 \mathrm{mg} \mathrm{ml}^{-1}$ was prepared by dissolving $25 \mathrm{mg}$ of EFO in $25 \mathrm{ml}$ of acetonitrile. From this solution, concentration in the range from $20 \mu \mathrm{g} \mathrm{ml}^{-1}$ to $120 \mu \mathrm{g} \mathrm{ml}^{-1}$ was prepared for linearity by dissolving $0.2,0.4,0.6,0.8,1.0$, and 1.2 $\mathrm{ml}$ of stock solution in $10 \mathrm{ml}$ of volumetric flask and making up to volume with the mobile phase (acetate buffer and acetonitrile 50:50).

\section{FORCED DEGRADATION STUDY AND PREPARATION OF SAMPLE SOLUTIONS}

Specificity of the method was determined by performing the forced degradation study of EFO by evaluating whether there is any impurity or degradation product is present in the analyte. EFO was subjected to hydrolytic, oxidative, thermal, and photolytic conditions as per the conditions prescribed by ICH guidelines. For the forced degradation study, initially, acetonitrile was added as a co-solvent but in alkaline degradation sample, the precipitate was obtained with acetonitrile so methanol was used as a 
co-solvent since EFO was insoluble in stressor reagents and water (Singh and Bakshi, 2000). EFO stock solutions $\left(1 \mathrm{mg} \mathrm{ml}^{-1}\right)$ were prepared in methanol. These stock solutions were diluted with 1 $\mathrm{M} \mathrm{HCl}, 0.5 \mathrm{M} \mathrm{NaOH}$, and $10 \%$ hydrogen peroxide $\left(\mathrm{H}_{2} \mathrm{O}_{2}\right)$ in the ratio $1: 1(\% \mathrm{v} / \mathrm{v})$. Effect of dry heat (thermal degradation) was performed on solid state. EFO was spread in a Petri dish and kept in an oven at $80^{\circ} \mathrm{C}$ for 11 days under dry heat conditions. During photodegradation, solid drug powder and solution form were exposed to fluorescent light (1.25 million lux hours) and UV light $\left(200 \mathrm{Whm}^{-2}\right)$ in a photostability chamber. All the degradation samples (acid, base degradation samples were neutralized with 1 $\mathrm{M} \mathrm{NaOH}$ and $0.5 \mathrm{M} \mathrm{HCl}$ ) were diluted to a concentration of $100 \mu \mathrm{g}$ $\mathrm{ml}^{-1}$ with the mobile phase (ammonium acetate buffer:acetonitrile $50: 50)$

\section{VALIDATION OF DEVELOPED METHOD (ICH Q2(R1), 2005 AND FDA, 2000)}

The method was developed as per ICH guidelines in terms of linearity, precision, limit of detection, limit of quantification, accuracy, and robustness.

System suitability tests were performed from six replicate injections of the drug solution. The result of each system suitability test was compared with the defined acceptance criteria. Retention factor, tailing factor $(\mathrm{T} \leq 2.0)$, theoretical plates $(\mathrm{N}>2,000)$, and \%RSD were evaluated for results. For linearity, solutions in the concentration ranging from $20 \mu \mathrm{g} \mathrm{ml}^{-1}$ to $120 \mu \mathrm{g}$ $\mathrm{ml}^{-1}$ were injected into the HPLC system. Linearity sample was injected in triplicate. Peak areas of the respective concentration were noted. Graph was prepared by plotting peak area versus concentration. Regression equation was obtained, and the value of $r^{2}$ was obtained from the regression equation. Precision was calculated by the repeatability method by evaluating in six different concentrations three times a day for intra-day precision and on three different days for inter-day precision. Twenty microliters of six different concentrations were injected, and the average of the peak areas and \%RSD was calculated. Accuracy was performed by the standard addition method. A known amount of EFO sample solution of a concentration of $40 \mu \mathrm{g} \mathrm{ml}^{-1}$ was added to the standard solutions to obtain concentrations of 60,80 , and $100 \mu \mathrm{g} \mathrm{ml}{ }^{-1}$. Recovery samples were prepared in triplicate and injected for analysis. Robustness was evaluated by analyzing deliberate changes in the method variables. The parameters taken for analysis were $\mathrm{pH}$ of buffer $(5.8 \pm 0.2$ units), initial gradient ratio $(2 \%)$, and flow rate $\left(1 \pm 0.1 \mathrm{ml} \mathrm{min}^{-1}\right)$.

\section{ISOLATION OF DEGRADATION PRODUCTS IN ALKALINE CONDITIONS}

Major degradation products formed were DP1, DP3, and DP4 in alkaline hydrolysis

Preparation of alkaline degradation samples $-1 \mathrm{~g}$ of EFO was added to $30 \mathrm{ml}$ of methanol and $20 \mathrm{ml}$ of $0.5 \mathrm{M} \mathrm{NaOH}$. The solution for degradation was maintained at room temperature for 48 hours. The solution was neutralized and analyzed by HPLC. In alkaline hydrolysis, DP1, DP3, and DP4 are formed with 50\%, $25 \%$, and $10 \%$, respectively, area by normalization.

Isolation of degraded samples by preparative HPLC For purification, degradation products DP1, DP3, and DP4 were separated by preparative HPLC. Fractions of greater than $97 \%$ were collected together. The solutions were concentrated on rotavapour to remove acetonitrile. To confirm the retention time of isolated impurity, isolated fraction was analyzed by HPLC. The solutions were dried in a lyophilizer. DP1, DP3, and DP4 were obtained as white solids.

\section{RESULTS AND DISCUSSION}

\section{HPLC method development}

To optimize the method, the trials were taken with water: methanol (50:50) and water: acetonitrile (50:50). Under these conditions, the peak was eluting late, and peak shape was broad with methanol. Other trials were taken with water: acetonitrile (35:65) and ammonium acetate buffer $\mathrm{pH}$ 5: acetonitrile. From this, ammonium acetate buffer $\mathrm{pH}$ 5: acetonitrile (35:65) was found suitable for optimization. During the development of the forced degradation study, degradation products were formed in alkaline conditions, and the degradation products (DP6-DP2) were co-eluting. In photolytic condition, one of the degradation products (DP8) was co-eluting with EFO peak. To separate the degradation products in alkaline degradation, $\mathrm{pH}$ range (3-6) was tried, and various trials with modification in the mobile phase ratio were tried in the isocratic phase with methanol. $\mathrm{pH} 5.8$ was found to be suitable for separation of degradation products in alkaline condition, and DP2 and DP3 peaks were merged with the addition of methanol in the mobile phase. For separation of DP8 from EFO in photolytic condition, various trials of the gradient were performed and also taken on C-8 column to separate DP8 from EFO. Finally, separation of DP8 from EFO and separation of degradation products (DP6-DP2) in alkaline conditions were achieved on $\mathrm{C}-18$ column with acetate buffer with $\mathrm{pH} 5.8$ and acetonitrile with the gradient program as (time/\% B) $0 / 25$, $6 / 25,11 / 40,35 / 50,45 / 50,50 / 53$, and 60/53. EFO was eluted at the retention time of 57.66 minutes (Fig. 1a). Various trials for separation of DP8 from EFO are shown in Table 1.

\section{Validation of the developed method}

System suitability testing is an integral part of the development method and was performed to evaluate the behavior

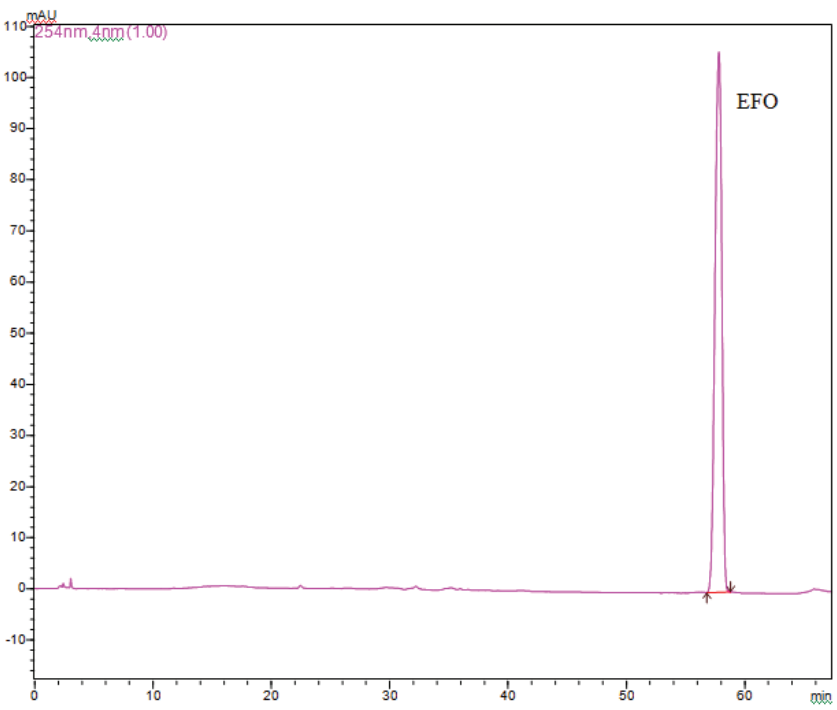

Figure 1a. Chromatogram of standard solution of EFO. 
Table 1. Trials for separation of DP8 from EFO.

\begin{tabular}{cll}
\hline S.No. & Mobile Phase & EFO and DP8 \\
\hline 1. & Gradient [Time (minutes)- \% ACN] - 0.01-25, 6-25, 11-40, 30-65, 50-65, 51-25, 55-STOP & EFO Rt- 34.8 minutes, EFO and DP8 were co-eluting \\
2. & Gradient [Time (minutes)- \% ACN] - 0.01-25, 6-25, 11-40, 25-65, 30-35. 40-35, 41-25, 45-STOP & EFO Rt- 35 minutes , EFO and DP8 were co-eluting \\
3. & Gradient [Time (minutes)- \% ACN]- 0.01-25, 6-25, 11-40, 22-40, 30-55, 45-55, 46-25, 50-STOP & EFO Rt- 44.1 minutes, EFO and DP8 were co-eluting \\
4. & Gradient [Time (minutes)- \% ACN]-0.01-25, 6-25, 11-40, 30-65, 50-65, 51-25, 55-STOP & EFO Rt- 34.6 minutes EFO and DP8 were co-eluting \\
\hline
\end{tabular}

of the chromatographic system. EFO eluted at 57.66 minutes. Tailing factor was less than 2 and theoretical plates were greater than 2,000. Linearity was calculated in the concentration range $20-120 \mu \mathrm{g} \mathrm{ml}^{-1}$. Regression coefficient $r^{2}$ was found to be 0.9994 with the regression equation $y=33223 x+15,744$. Based on the signal-to-noise ratio, the limit of detection was found to be 0.41 $\mu \mathrm{g} \mathrm{ml} \mathrm{m}^{-1}$, and the limit of quantification was found to be 1.24 $\mu \mathrm{g} \mathrm{ml}^{-1}$. Intraday and interday precision were calculated at six concentration levels $20-120 \mu \mathrm{g} \mathrm{ml}^{-1}$. \%RSD values of intraday and interday precision were found to be less than 2 . The developed method was found to be precise. Recovery studies were performed by the standard addition method. \% Recovery was found to be in the range of $99.7-100.25 \%$. The developed method was accurate. Robustness was performed by analyzing slight changes in the method variables. The study was evaluated by changing the $\mathrm{pH}$ of buffer (5.8 \pm 0.2 units), initial gradient ratio ( $2 \%)$, and flow rate $\left(1 \pm 0.1 \mathrm{ml} \mathrm{min}^{-1}\right)$ at a concentration of $40 \mu \mathrm{g} \mathrm{ml}^{-1}$. The developed method was robust for all parameters. The results of system suitability parameters and validation parameters are shown in Table 2.

\section{Forced degradation study}

No degradation was observed when $\mathrm{EFO}$ was subjected to $1 \mathrm{M} \mathrm{HCl}$ at $80^{\circ} \mathrm{C}$ for 5 hours, $10 \%$ hydrogen peroxide at $\mathrm{RT}$ for 24 hours and dry heat at $80^{\circ} \mathrm{C}$ for 11 days. EFO when subjected to $0.5 \mathrm{M} \mathrm{NaOH}$ at room temperature for 6 hours, significant degradation $(44.18 \%)$ was observed. There was a formation of six degradation products such as DP1 (28.14\%), DP2 (4.01\%), DP3 (8.8\%), DP4 (2.17\%), DP5 (0.66\%), and DP6 $(0.63 \%)$ at the retention time of 52.91 minutes, 26.66 minutes, 24.09 minutes, 20.29 minutes, 18.83 minutes, and 14.33 minutes (Fig. 1b). Slight degradation was observed in EFO and subjected to solution form in photolytic condition (11.6\%) with the formation of degradation products DP10 (1.7\%), DP9 $(0.48 \%)$, DP8 $(8.1 \%)$, and DP7 $(0.98 \%)$ minutes at the retention time of 11.67 minutes, 30.01 minutes, 55.92 minutes, and 31.9 minutes, respectively (Fig. 1c).

From the above, it was observed that EFO was labile to alkaline and photolytic conditions in solution form, whereas it was stable in acidic, oxidative, and photolytic conditions in solid state.

\section{Identification of degradation products by LC-Q-TOF-MS}

Degradation products formed in alkaline and photolytic conditions were identified by LC-ESI-Q-TOF-MS. Highresolution mass corresponding to the elemental composition of EFO and its DPs are shown in Table 3.

$\mathrm{EFO}-\mathrm{EFO}$ is eluted at the retention time of 57.1 minutes. Molecular mass of Efonidipine $\mathrm{HCl}$ ethanolate is $\mathrm{C}_{36} \mathrm{H}_{45} \mathrm{ClN}_{3} \mathrm{O}_{8} \mathrm{P}$. EFO shows a corresponding mass of the Efonidipine base. Masses of $\mathrm{HCl}$ and Ethanolate are absent in mass spectra since their mass values are less than $50 \mathrm{~m} / \mathrm{z}$ and ethanol is volatile so its mass is absent in mass spectra. The mass spectra of EFO show protonated
Table 2. System suitability and validation parameters of EFO.

\begin{tabular}{|c|c|c|c|}
\hline \multicolumn{2}{|c|}{ System suitability Parameters } & \multicolumn{2}{|c|}{ Value } \\
\hline \multicolumn{2}{|c|}{ Retention Time (minutes $\pm \mathrm{SD}^{\mathrm{a}}$ ) } & \multicolumn{2}{|c|}{$57.66 \pm 0.05$} \\
\hline \multicolumn{2}{|c|}{ Tailing factor $\pm \mathrm{SD}^{\mathrm{a}}$} & \multicolumn{2}{|c|}{$0.94 \pm 0.007$} \\
\hline \multicolumn{2}{|l|}{ Theoretical Plates $\pm \mathrm{SD}^{\mathrm{a}}$} & \multicolumn{2}{|c|}{$69,040 \pm 424.06$} \\
\hline \multicolumn{2}{|l|}{ Validation Parameters } & \multicolumn{2}{|c|}{ Value } \\
\hline \multicolumn{2}{|l|}{ Calibration range } & \multicolumn{2}{|c|}{$20-120 \mu \mathrm{g} \mathrm{ml}^{-1}$} \\
\hline \multicolumn{2}{|l|}{$\operatorname{LOD}\left(\mu \mathrm{g} \mathrm{ml}^{-1}\right)$} & \multicolumn{2}{|c|}{0.41} \\
\hline \multicolumn{2}{|l|}{$\operatorname{LOQ}\left(\mu \mathrm{g} \mathrm{ml}^{-1}\right)$} & \multicolumn{2}{|c|}{1.24} \\
\hline \multicolumn{2}{|l|}{ Regression Equation } & \multicolumn{2}{|c|}{$y=33223 x+15744$} \\
\hline \multicolumn{2}{|l|}{ Correlation coefficient } & \multicolumn{2}{|c|}{0.999} \\
\hline \multicolumn{2}{|l|}{ Accuracy } & \multicolumn{2}{|c|}{$\%$ Recovery (Mean $\pm \mathrm{SD}^{\mathrm{a}}$ ) } \\
\hline \multicolumn{2}{|l|}{$50 \%$} & \multicolumn{2}{|c|}{$100.16 \pm 0.28$} \\
\hline \multicolumn{2}{|l|}{$100 \%$} & \multicolumn{2}{|c|}{$100.25 \pm 0.25$} \\
\hline \multicolumn{2}{|l|}{$150 \%$} & \multicolumn{2}{|c|}{$99.77 \pm 0.09$} \\
\hline \multicolumn{2}{|l|}{ Precision } & \multicolumn{2}{|c|}{$\% \mathrm{RSD}^{\mathrm{b}}$} \\
\hline \multicolumn{2}{|l|}{ Intraday } & \multicolumn{2}{|c|}{1.20} \\
\hline \multicolumn{2}{|l|}{ Interday } & & \\
\hline Robustness & & & \\
\hline Parameter & Levels & $\% \mathrm{RSD}(\mathrm{Rt})$ & $\% \mathrm{RSD}^{\mathrm{b}}$ (Area) \\
\hline $\mathrm{pH}$ & 5.6 & 0.87 & 0.15 \\
\hline & 5.8 & 0.72 & 0.07 \\
\hline & 6.0 & 0.75 & 0.18 \\
\hline Initial gradient ratio & 23 & 0.37 & 0.14 \\
\hline & 25 & 0.38 & 0.20 \\
\hline & 27 & 0.48 & 0.10 \\
\hline Flow rate & 0.9 & 0.51 & 0.12 \\
\hline & 1.0 & 0.37 & 0.04 \\
\hline & 1.1 & 0.39 & 0.03 \\
\hline
\end{tabular}

$\mathrm{a}=$ Standard Deviation, $\mathrm{b}=\%$ Relative Standard Deviation

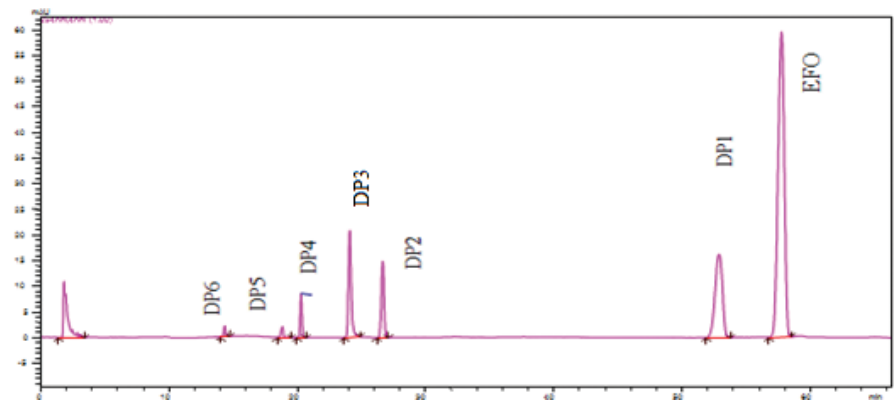

Figure 1b. Chromatogram of alkaline degradation of EFO.

molecular ion at $\mathrm{m} / \mathrm{z} 632$ corresponds to molecular formula $\mathrm{C}_{34} \mathrm{H}_{38} \mathrm{~N}_{3} \mathrm{O}_{7} \mathrm{P}$. EFO undergoes fragmentation at $\mathrm{m} / z 562$ (loss of $\mathrm{C}_{4} \mathrm{H}_{5} \mathrm{O}$ from $\mathrm{m} / \mathrm{z}$ 632), 449 (loss of N-benzyl amino group from $\mathrm{m} / \mathrm{z}$ 632), 405 (loss of ethoxy group from $\mathrm{m} / \mathrm{z} 449$ ), 337 (loss of 


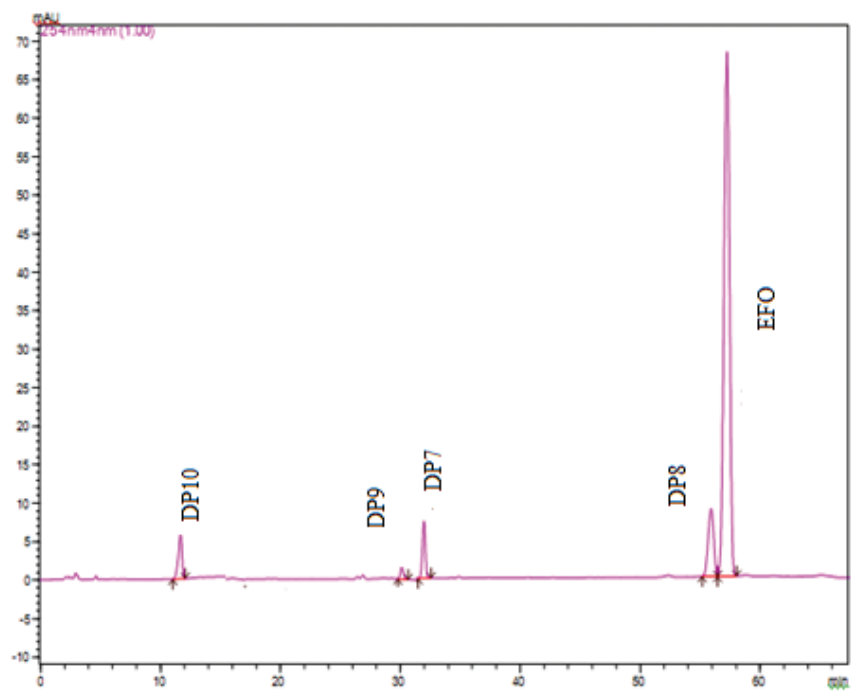

Figure 1c. Chromatogram of photolytic degradation (solution) of EFO.
$\mathrm{C}_{5} \mathrm{H}_{8}$ from $\mathrm{m} / z$ 405), and 210 (loss of $\mathrm{C}_{19} \mathrm{H}_{22} \mathrm{~N}_{2} \mathrm{O}_{7} \mathrm{P}^{+}$from $\mathrm{m} / \mathrm{z} 632$ ) (Supple Figure S1a and b).

DP1 - DP1 is eluted at the retention time of 52.91 minutes. LC-ESI/MS/MS spectrum of DP1 shows protonated molecular ion at $m / z 664$ with the elemental composition of $\mathrm{C}_{35} \mathrm{H}_{42} \mathrm{~N}_{3} \mathrm{O}_{8} \mathrm{P}^{+}$. DP1 undergoes fragmentation to produce ions at $m / z 608$ (loss of $\mathrm{C}_{3} \mathrm{H}_{3} \mathrm{O}$ from 664), $m / z$ 481(loss of $\mathrm{C}_{13} \mathrm{H}_{12} \mathrm{~N}$ from $\mathrm{m} / \mathrm{z}$ 664), $\mathrm{m} / \mathrm{z} 437$ (loss of $\mathrm{C}_{6} \mathrm{H}_{13} \mathrm{NO}_{6} \mathrm{P}$ from 664), $\mathrm{m} / z$ 351(loss of $\mathrm{C}_{17} \mathrm{H}_{23} \mathrm{O}$ from $\mathrm{m} / z$ 608), $\mathrm{m} / \mathrm{z}$ 269 (loss of $\mathrm{C}_{16} \mathrm{H}_{23} \mathrm{~N}_{2} \mathrm{O}_{4} \mathrm{P}^{+}$from 608), $m / z 210$ (loss of $\mathrm{C}_{14} \mathrm{H}_{13} \mathrm{NO}_{2}^{+}$ from $\mathrm{m} / \mathrm{z} 437$ ), and $\mathrm{m} / \mathrm{z} 181$ (loss of $\mathrm{C}_{2} \mathrm{H}_{5}$ from $\mathrm{m} / \mathrm{z} 210$ ). DP1 formed by ring-opening of phosphinane and esterification by cosolvent methanol (Supple Figure S2a and b).

DP2 - DP2 is eluted at the retention time of 26.66 minutes. DP2 is formed with protonated molecular ion $\mathrm{m} / \mathrm{z} 650$ with the elemental composition of $\mathrm{C}_{34} \mathrm{H}_{41} \mathrm{~N}_{3} \mathrm{O}_{8} \mathrm{P}^{+}$. This mass is formed by ring-opening of phosphinane group (Supple Figure S3a).

DP3 - DP3 is eluted at the retention time of 24.06 minutes. DP3 is formed with protonated molecular ion $\mathrm{m} / \mathrm{z}$ 228 with the elemental composition of $\mathrm{C}_{15} \mathrm{H}_{18} \mathrm{NO}^{+}$. DP3 shows

Table 3. High-resolution mass data corresponding to the elemental composition of $[\mathrm{M}+\mathrm{H}]^{+}$of EFO and its DPs.

\begin{tabular}{|c|c|c|c|c|c|}
\hline Description & Retention Time & Molecular Formula & Observed Mass & Calculated Mass & Difference (ppm) \\
\hline \multirow{7}{*}{ EFO } & \multirow{7}{*}{57.33 minutes } & $\mathrm{C}_{34} \mathrm{H}_{38} \mathrm{~N}_{3} \mathrm{O}_{7} \mathrm{P}^{+}$ & 632.2528 & 632.2514 & 1.4 \\
\hline & & $\mathrm{C}_{30} \mathrm{H}_{33} \mathrm{~N}_{3} \mathrm{O}_{6} \mathrm{P}^{+}$ & 562.3946 & 562.3101 & -8.45 \\
\hline & & $\mathrm{C}_{27} \mathrm{H}_{32} \mathrm{~N}_{2} \mathrm{O}_{5} \mathrm{P}^{+}$ & 495.1718 & 495.2043 & 3.25 \\
\hline & & $\mathrm{C}_{19} \mathrm{H}_{22} \mathrm{~N}_{2} \mathrm{O}_{6} \mathrm{P}^{+}$ & 405.1172 & 405.122 & -4.8 \\
\hline & & $\mathrm{C}_{21} \mathrm{H}_{26} \mathrm{~N}_{2} \mathrm{O}_{7} \mathrm{P}^{+}$ & 449.143 & 449.1474 & -4.4 \\
\hline & & $\mathrm{C}_{14} \mathrm{H}_{14} \mathrm{~N}_{2} \mathrm{O}_{6} \mathrm{P}^{+}$ & 337.0544 & 337.0584 & -4 \\
\hline & & $\mathrm{C}_{15} \mathrm{H}_{16} \mathrm{~N}^{+}$ & 210.126 & 210.1277 & -1.7 \\
\hline \multirow{8}{*}{ DP1 } & \multirow{8}{*}{52.91 minutes } & $\mathrm{C}_{35} \mathrm{H}_{42} \mathrm{~N}_{3} \mathrm{O}_{8} \mathrm{P}+$ & 664.278 & 664.2743 & -0.37 \\
\hline & & $\mathrm{C}_{32} \mathrm{H}_{39} \mathrm{~N}_{3} \mathrm{O}_{7} \mathrm{P}^{+}$ & 608.3383 & 608.252 & -8.63 \\
\hline & & $\mathrm{C}_{22} \mathrm{H}_{30} \mathrm{~N}_{2} \mathrm{O}_{8} \mathrm{P}^{+}$ & 481.1701 & 481.1734 & 0.33 \\
\hline & & $\mathrm{C}_{29} \mathrm{H}_{29} \mathrm{~N}_{2} \mathrm{O}_{2}^{+}$ & 437.144 & 437.1424 & -0.16 \\
\hline & & $\mathrm{C}_{15} \mathrm{H}_{16} \mathrm{~N}_{2} \mathrm{O}_{6} \mathrm{P}+$ & 351.0706 & 351.0778 & 0.72 \\
\hline & & $\mathrm{C}_{16} \mathrm{H}_{16} \mathrm{NO}_{3}^{+}$ & 269.9899 & 270.1125 & 12.26 \\
\hline & & $\mathrm{C}_{15} \mathrm{H}_{16} \mathrm{~N}^{+}$ & 210.125 & 210.1277 & 0.27 \\
\hline & & $\mathrm{C}_{13} \mathrm{H}_{11} \mathrm{~N}$ & 181.0745 & 181.0891 & 1.46 \\
\hline \multirow[t]{4}{*}{ DP2 } & \multirow[t]{2}{*}{26.66 minutes } & $\mathrm{C}_{34} \mathrm{H}_{40} \mathrm{~N}_{3} \mathrm{O}_{8} \mathrm{P}^{+}$ & 650.2543 & 650.2587 & -0.44 \\
\hline & & $\mathrm{C}_{15} \mathrm{H}_{17} \mathrm{NO}^{+}$ & 228.1373 & 228.1344 & 2.9 \\
\hline & \multirow{6}{*}{24.09 minutes } & $\mathrm{C}_{15} \mathrm{H}_{15} \mathrm{~N}^{+}$ & 209.0322 & 209.0204 & 11.8 \\
\hline & & $\mathrm{C}_{13} \mathrm{H}_{10} \mathrm{~N}^{+}$ & 180.066 & 180.0708 & -4.8 \\
\hline \multirow[t]{4}{*}{ DP3 } & & $\mathrm{C}_{11} \mathrm{H}_{14} \mathrm{~N}^{+}$ & 160.1093 & 160.1121 & -2.8 \\
\hline & & $\mathrm{C}_{8} \mathrm{H}_{12} \mathrm{~N}^{+}$ & 122.0959 & 122.0964 & -0.5 \\
\hline & & $\mathrm{C}_{7} \mathrm{H}_{4} \mathrm{~N}^{+}$ & 102.1275 & 102.1338 & -6.3 \\
\hline & & $\mathrm{C}_{4} \mathrm{H}_{8} \mathrm{NO}^{+}$ & 86.0965 & 86.0912 & 5.3 \\
\hline \multirow{6}{*}{ DP4 } & \multirow{6}{*}{20.29 minutes } & $\mathrm{C}_{21} \mathrm{H}_{29} \mathrm{~N}_{2} \mathrm{O}_{8} \mathrm{P}^{+}$ & 469.1705 & 469.17 & -0.05 \\
\hline & & $\mathrm{C}_{20} \mathrm{H}_{27} \mathrm{~N}_{2} \mathrm{O}_{6} \mathrm{P}$ & 422.0636 & 422.1607 & 9.71 \\
\hline & & $\mathrm{C}_{15} \mathrm{H}_{16} \mathrm{~N}_{2} \mathrm{O}_{6} \mathrm{P}^{+}$ & 351.0698 & 351.071 & 0.12 \\
\hline & & $\mathrm{C}_{13} \mathrm{H}_{11} \mathrm{~N}_{2} \mathrm{O}_{3}^{+}$ & 243.002 & 243.074 & 7.2 \\
\hline & & $\mathrm{C}_{15} \mathrm{H}_{15} \mathrm{~N}_{2} \mathrm{O}_{3}^{+}$ & 271.008 & 271.1077 & 9.97 \\
\hline & & $\mathrm{C}_{6} \mathrm{H}_{14} \mathrm{O}_{4} \mathrm{P}^{+}$ & 181.074 & 181.0624 & -1.16 \\
\hline
\end{tabular}




\begin{tabular}{|c|c|c|c|c|c|}
\hline \multicolumn{6}{|c|}{ Table 3. (Continued) } \\
\hline Description & Retention Time & Molecular Formula & Observed Mass & Calculated Mass & Difference (ppm) \\
\hline \multirow{10}{*}{ DP5 } & \multirow{10}{*}{18.83 minutes } & $\mathrm{C}_{20} \mathrm{H}_{26} \mathrm{~N}_{2} \mathrm{O}_{7} \mathrm{P}+$ & 437.1454 & 437.1433 & -0.21 \\
\hline & & $\mathrm{C}_{19} \mathrm{H}_{22} \mathrm{~N}_{2} \mathrm{O}_{6} \mathrm{P}+$ & 405.1162 & 405.122 & 0.58 \\
\hline & & $\mathrm{C}_{19} \mathrm{H}_{23} \mathrm{NO}_{4} \mathrm{P}^{+}$ & 360.1312 & 360.1359 & 0.47 \\
\hline & & $\mathrm{C}_{17} \mathrm{H}_{22} \mathrm{NO}_{3} \mathrm{P}^{+}$ & 319.0424 & 319.0521 & 0.97 \\
\hline & & $\mathrm{C}_{17} \mathrm{H}_{19} \mathrm{O}_{3} \mathrm{P}^{+}$ & 302.0366 & 302.1072 & 7.06 \\
\hline & & $\mathrm{C}_{19} \mathrm{H}_{21} \mathrm{~N}_{2} \mathrm{O}_{5} \mathrm{P}^{+}$ & 388.1206 & 388.1188 & -0.18 \\
\hline & & $\mathrm{C}_{14} \mathrm{H}_{12} \mathrm{NO}_{2} \mathrm{P}^{+}$ & 257.0086 & 257.0606 & 5.2 \\
\hline & & $\mathrm{C}_{11} \mathrm{H}_{13} \mathrm{NO}_{3} \mathrm{P}^{+}$ & 238.0012 & 238.0628 & 6.16 \\
\hline & & $\mathrm{C}_{11} \mathrm{H}_{10} \mathrm{NOP}^{+}$ & 203.0584 & 203.05 & -0.84 \\
\hline & & $\mathrm{C}_{9} \mathrm{H}_{10} \mathrm{OP}^{+}$ & 164.9179 & 165.0464 & 12.85 \\
\hline \multirow{3}{*}{ DP6 } & \multirow{3}{*}{14.33 minutes } & $\mathrm{C}_{7} \mathrm{H}_{2} \mathrm{OP}$ & 136.1128 & 136.0078 & -10.5 \\
\hline & & $\mathrm{C}_{20} \mathrm{H}_{28} \mathrm{~N}_{2} \mathrm{O}_{8} \mathrm{P}^{+}$ & 455.2 & 455.1505 & -4.95 \\
\hline & & $\mathrm{C}_{27} \mathrm{H}_{31} \mathrm{~N}_{3} \mathrm{O}_{7} \mathrm{P}^{+}$ & 540.1858 & 540.1855 & 0.55 \\
\hline \multirow{8}{*}{ DP7 } & \multirow{7}{*}{31.7 minutes } & $\mathrm{C}_{26} \mathrm{H}_{29} \mathrm{~N}_{3} \mathrm{O}_{6} \mathrm{P}^{+}$ & 509.2001 & 509.1716 & -2.85 \\
\hline & & $\mathrm{C}_{18} \mathrm{H}_{21} \mathrm{~N}_{2} \mathrm{O}_{4} \mathrm{P}^{+}$ & 360.0288 & 360.1239 & 9.51 \\
\hline & & $\mathrm{C}_{8} \mathrm{H}_{10} \mathrm{~N}^{+}$ & 120.0793 & 120.0808 & 0.15 \\
\hline & & $\mathrm{C}_{10} \mathrm{H}_{12} \mathrm{~N}^{+}$ & 145.0658 & 145.0891 & 2.33 \\
\hline & & $\mathrm{C}_{21} \mathrm{H}_{24} \mathrm{~N}_{2} \mathrm{O}_{7} \mathrm{P}^{+}$ & 447.131 & 447.1399 & -6.4 \\
\hline & & $\mathrm{C}_{19} \mathrm{H}_{21} \mathrm{~N}_{2} \mathrm{O}_{7} \mathrm{P}^{+}$ & 421.1114 & 421.112 & -2.3 \\
\hline & & $\mathrm{C}_{13} \mathrm{H}_{17} \mathrm{NO}_{4} \mathrm{P}^{+}$ & 283.0462 & 283.0477 & -5.2 \\
\hline & \multirow{11}{*}{55.92 minutes } & $\mathrm{C}_{8} \mathrm{H}_{13} \mathrm{NO}_{3} \mathrm{P}^{+}$ & 202.1781 & 202.1628 & -1.53 \\
\hline \multirow{10}{*}{ DP8 } & & $\mathrm{C}_{34} \mathrm{H}_{37} \mathrm{~N}_{3} \mathrm{O}_{7} \mathrm{P}^{+}$ & 630.23 & 630.2324 & -3.8 \\
\hline & & $\mathrm{C}_{32} \mathrm{H}_{33} \mathrm{~N}_{3} \mathrm{O}_{6} \mathrm{P}^{+}$ & 586.2371 & 586.238 & -1.53 \\
\hline & & $\mathrm{C}_{29} \mathrm{H}_{26} \mathrm{~N}_{3} \mathrm{O}_{4} \mathrm{P}^{+}$ & 511.1617 & 511.1661 & 0.44 \\
\hline & & $\mathrm{C}_{21} \mathrm{H}_{24} \mathrm{~N}_{2} \mathrm{O}_{7} \mathrm{P}^{+}$ & 447.128 & 447.1316 & -3.47 \\
\hline & & $\mathrm{C}_{19} \mathrm{H}_{20} \mathrm{~N}_{2} \mathrm{O}_{6} \mathrm{P}^{+}$ & 403.1012 & 403.1026 & -3.2 \\
\hline & & $\mathrm{C}_{19} \mathrm{H}_{22} \mathrm{~N}_{2} \mathrm{O}_{4} \mathrm{P}^{+}$ & 373.1349 & 373.1312 & -0.37 \\
\hline & & $\mathrm{C}_{16} \mathrm{H}_{20} \mathrm{~N}_{2} \mathrm{O}_{4} \mathrm{P}^{+}$ & 335.0391 & 335.0402 & -3.68 \\
\hline & & $\mathrm{C}_{16} \mathrm{H}_{19} \mathrm{~N}_{2} \mathrm{O}_{2}^{+}$ & 271.0699 & 271.0709 & 2.8 \\
\hline & & $\mathrm{C}_{15} \mathrm{H}_{16} \mathrm{~N}^{+}$ & 210.1254 & 210.1244 & 1.1 \\
\hline & & $\mathrm{C}_{7} \mathrm{H}_{7}^{+}$ & 91.0534 & 91.0542 & 0.08 \\
\hline \multirow{4}{*}{ DP9 } & \multirow{4}{*}{30.01 minutes } & $\mathrm{C}_{27} \mathrm{H}_{33} \mathrm{~N}_{3} \mathrm{O}_{7} \mathrm{P}^{+}$ & 542.2017 & 542.2011 & -7.6 \\
\hline & & $\mathrm{C}_{19} \mathrm{H}_{22} \mathrm{~N}_{2} \mathrm{O}_{6} \mathrm{P}^{+}$ & 405.1167 & 405.121 & -2.82 \\
\hline & & $\mathrm{C}_{17} \mathrm{H}_{22} \mathrm{NO}_{3} \mathrm{P}^{+}$ & 319.0484 & 319.0493 & 0.09 \\
\hline & & $\mathrm{C}_{7} \mathrm{H}_{6} \mathrm{~N}^{+}$ & 104.1087 & 104.0495 & -5.92 \\
\hline DP10 & 11.67 minutes & $\mathrm{C}_{28} \mathrm{H}_{35} \mathrm{~N}_{2} \mathrm{O}_{5} \mathrm{P}^{+}$ & 510.212 & 510.2284 & 3.93 \\
\hline
\end{tabular}

fragments of $m / z 209$ (loss of hydronium ion from $\mathrm{m} / \mathrm{z} 228$ ), $\mathrm{m} / \mathrm{z}$ 180 (loss of ethyl group from $\mathrm{m} / z$ 209), $\mathrm{m} / z 160$ (loss of $\mathrm{C}_{4} \mathrm{H}$ from $m / z$ 209), $m / z 122$ (loss of $\mathrm{C}_{7} \mathrm{H}_{6} \mathrm{O}$ from $\mathrm{m} / z$ 228), $\mathrm{m} / z 102$ (loss of $\mathrm{C}_{6} \mathrm{H}_{6}$ from $\mathrm{m} / \mathrm{z} 180$ ), and $\mathrm{m} / \mathrm{z} 86$ (loss of $\mathrm{C}_{11} \mathrm{H}_{10}$ from $\mathrm{m} / \mathrm{z} 228$ ). DP3 is formed by hydrolysis at ester group of EFO with the loss of aromatic nitro group, 1, 4 dihydropyridine and cyclic phosphinane ring (Supple Figure S4a and b).

DP4 - DP4 is eluted at the retention time of 20.29 minutes. LC-ESI/MS/MS spectrum of DP4 shows protonated molecular ion peak at $\mathrm{m} / \mathrm{z} 469$ with the elemental composition of $\mathrm{C}_{21} \mathrm{H}_{29} \mathrm{~N}_{2} \mathrm{O}_{8} \mathrm{P}^{+}$. DP4 shows fragment ions of $m / z 422$ (loss of $\mathrm{CH}_{2} \mathrm{O}_{2}$ ) and $m / z 271$ (loss of $\mathrm{C}_{6} \mathrm{H}_{14} \mathrm{O}_{5} \mathrm{P}^{+}$). Fragment ion $\mathrm{m} / z 422$ undergoes further fragmentation to produce ions at $\mathrm{m} / \mathrm{z} 351$ (loss of
$\mathrm{C}_{5} \mathrm{H}_{11}$ from $m / z$ 422), $m / z 243$ (loss of $\mathrm{C}_{2} \mathrm{H}_{5} \mathrm{O}_{3} \mathrm{P}$ from 351), and $m / z$ 181 (loss of $\mathrm{C}_{14} \mathrm{H}_{13} \mathrm{~N}_{2} \mathrm{O}_{2}$ from $m / z$ 422) (Supple Figure $\mathrm{S} 5 \mathrm{a}$ and $\mathrm{b}$ ). $\mathrm{DP} 5-\mathrm{DP} 5$ is eluted at the retention time of 18.83 minutes. LC-ESI/MS/MS spectrum of DP5 shows protonated molecular ion peak at $\mathrm{m} / \mathrm{z} 437$ with the elemental composition of $\mathrm{C}_{20} \mathrm{H}_{26} \mathrm{~N}_{2} \mathrm{O}_{7} \mathrm{P}^{+}$. DP5 shows fragment ions of $\mathrm{m} / \mathrm{z} 405$ (loss of methoxy group from $\mathrm{m} / z$ 437), and $\mathrm{m} / \mathrm{z} 388$ (loss of from $\mathrm{CH}_{5} \mathrm{O}_{2}$ $\mathrm{m} / \mathrm{z} 437) . \mathrm{m} / \mathrm{z} 405$ undergoes further fragmentation to produce ions of $\mathrm{m} / \mathrm{z} 360$ (loss of nitrous acid from $\mathrm{m} / \mathrm{z} 405$ ), $\mathrm{m} / \mathrm{z} 319$ (loss of $\mathrm{C}_{2} \mathrm{HO}$ from $\mathrm{m} / \mathrm{z} 360$ ), $\mathrm{m} / \mathrm{z} 302$ (loss of $\mathrm{NH}_{3}$ from $\mathrm{m} / \mathrm{z} 319$ ). Further fragmentation takes place at $\mathrm{m} / \mathrm{z} 388$ to produce ions of $\mathrm{m} / \mathrm{z}$ 257 (loss of $\mathrm{C}_{5} \mathrm{H}_{9} \mathrm{NO}_{3}$ from $\mathrm{m} / z$ 388), $\mathrm{m} / \mathrm{z} 238$ (loss of $\mathrm{C}_{8} \mathrm{H}_{8} \mathrm{NO}_{2}$ from $\mathrm{m} / \mathrm{z}$ 388), $\mathrm{m} / \mathrm{z} 203$ (loss of $\mathrm{H}_{3} \mathrm{O}_{2}$ from 238), $\mathrm{m} / \mathrm{z} 164$ (loss 
of $\mathrm{C}_{2} \mathrm{~N}$ from $\mathrm{m} / \mathrm{z} 203$ ), and $\mathrm{m} / \mathrm{z} 136$ (loss of $\mathrm{C}_{2} \mathrm{H}_{5}$ from $\mathrm{m} / \mathrm{z} 164$ ) (Supple Figure S6a and b).

DP6 - DP6 is eluted at the retention time of 14.33 minutes. DP6 is formed with protonated molecular ion at $\mathrm{m} / \mathrm{z} 455$ with the elemental composition of $\mathrm{C}_{20} \mathrm{H}_{28} \mathrm{~N}_{2} \mathrm{O}_{8} \mathrm{P}^{+}$. It undergoes fragmentation by removal of methyl group to give $\mathrm{m} / \mathrm{z}$ at 441 (Supple Figure S7a and b).

DP7 - DP7 is eluted at the retention time of 31.9 minutes. LC-ESI/MS/MS spectrum of DP7 shows protonated molecular ion peak at $m / z 540$ with the elemental composition of $\mathrm{C}_{27} \mathrm{H}_{31} \mathrm{~N}_{3} \mathrm{O}_{7} \mathrm{P}^{+}$. DP7 shows fragment ions of $\mathrm{m} / z 509$ (loss of $\mathrm{CH}_{2} \mathrm{O}$ from $\mathrm{m} / z$ 540) and $m / z 447$ (loss of $\mathrm{C}_{6} \mathrm{H}_{7} \mathrm{~N}$ from $\mathrm{m} / z$ 540). Further fragmentation of $\mathrm{m} / z 509$ gives to ions of $\mathrm{m} / \mathrm{z} 360$ (loss of $\mathrm{C}_{8} \mathrm{H}_{8} \mathrm{NO}_{2}$ from $\mathrm{m} / \mathrm{z}$ 540), $m / z 120$ (loss of $\mathrm{C}_{8} \mathrm{H}_{8} \mathrm{NO}_{2}$ from $\mathrm{m} / z$ 509), and $\mathrm{m} / z 145$ (loss of $\mathrm{C}_{8} \mathrm{H}_{9} \mathrm{NO}_{4} \mathrm{P}$ from $\mathrm{m} / z$ 360). Fragmentation of $\mathrm{m} / z 447$ gives ions at $m / z 421$ (loss of $\mathrm{C}_{2} \mathrm{H}_{3}$ from $m / z 447$ ), $m / z 283$ (loss of $\mathrm{C}_{6} \mathrm{H}_{4} \mathrm{NO}_{3}$ from $\mathrm{m} / z$ 421), and $\mathrm{m} / z 202$ (loss of $\mathrm{C}_{5} \mathrm{H}_{4} \mathrm{O}$ from $\mathrm{m} / z$ 283). DP7 is formed by the loss of benzyl group from EFO (Supple Figure S8a and b).

DP8 - DP8 is eluted at the retention time of 55.92 minutes. LC-ESI/MS/MS spectrum of DP8 shows protonated molecular ion peak at $\mathrm{m} / \mathrm{z} 630$ with the elemental composition of $\mathrm{C}_{27} \mathrm{H}_{33} \mathrm{~N}_{3} \mathrm{O}_{7} \mathrm{P}^{+}$. DP8 shows protonated fragment ion at $\mathrm{m} / z 586$ (loss of $\mathrm{C}_{2} \mathrm{H}_{4} \mathrm{O}$ from $\mathrm{m} / \mathrm{z}$ 630). This fragment undergoes further fragmentation at $\mathrm{m} / \mathrm{z} 511$ (loss of $\mathrm{C}_{3} \mathrm{H}_{7} \mathrm{O}_{2}$ ) and at $\mathrm{m} / \mathrm{z} 373$ (loss of $\mathrm{C}_{13} \mathrm{H}_{11} \mathrm{NO}_{2}$ ). Fragment ion $\mathrm{m} / \mathrm{z} 373$ undergoes further fragmentation at $\mathrm{m} / \mathrm{z} 335$ (loss of $\mathrm{C}_{3} \mathrm{H}_{2}$ from $\mathrm{m} / \mathrm{z} 373$ ) and $\mathrm{m} / \mathrm{z} 271$ (loss of meta phosphoric acid from $\mathrm{m} / \mathrm{z} 335$ ). DP8 undergoes fragmentation to produce fragment ion at $\mathrm{m} / z 447$ (loss of $\mathrm{C}_{13} \mathrm{H}_{13} \mathrm{~N}$ ), $\mathrm{m} / z 403$ (loss of $\mathrm{C}_{2} \mathrm{H}_{4} \mathrm{O}$ from 447), $\mathrm{m} / z 210$ (loss of $\mathrm{C}_{19} \mathrm{H}_{21} \mathrm{~N}_{2} \mathrm{O}_{7} \mathrm{P}$ from $\mathrm{m} / z$ 630), and $m / z 91$ (loss of $\mathrm{C}_{8} \mathrm{H}_{9} \mathrm{~N}$ from $\mathrm{m} / z 210$ ). DP8 is formed from EFO by dehydrogenation at a protonated $\mathrm{m} / \mathrm{z}$ value of 630.23 (Supple Figure S9a and b).
DP9-DP9 is eluted at the retention time of 30.01 minutes LC-ESI/MS/MS spectrum of DP9 shows protonated molecular ion peak at $m / z 542$ with the elemental composition of $\mathrm{C}_{27} \mathrm{H}_{33} \mathrm{~N}_{3} \mathrm{O}_{7} \mathrm{P}^{+}$. DP9 shows fragment ions of $\mathrm{m} / z 405$ (loss of $\mathrm{C}_{8} \mathrm{H}_{11} \mathrm{NOP}$ from $\mathrm{m} / z$ 542), $\mathrm{m} / z 319$ (loss of $\mathrm{C}_{2} \mathrm{NO}_{3}$ from $\mathrm{m} / \mathrm{z} 405$ ), and $\mathrm{m} / z 104$ (loss of $\mathrm{C}_{10} \mathrm{H}_{16} \mathrm{O}_{3} \mathrm{P}$ from $\mathrm{m} / \mathrm{z} 542$ ). DP9 is formed by the loss of phenyl ethoxy amino group (Supple Figure S10a and b).

DP10 - DP10 is eluted at the retention time of 11.67 minutes and formed at $\mathrm{m} / \mathrm{z} 510$ with the elemental composition of $\mathrm{C}_{28} \mathrm{H}_{35} \mathrm{~N}_{2} \mathrm{O}_{5} \mathrm{P}^{+}$. DP10 is formed from the EFO by the removal of nitrophenyl group (Supple Figure S11a).

\section{Degradation pathway of EFO}

Alkaline condition - EFO possesses ester functional group and phosphinane ring. EFO in alkaline condition with the hydrolysis of phosphinane ring forms DP2. Hydrolysis of DP2 at phosphinane ring as a result there is ring-opening of phosphinane ring, and esterification with co-solvent methanol forms pseudo degradation product DP1, which on further hydrolysis at ester functional group forms pseudo degradation product DP4. DP4 on further hydrolysis at ester functional group forms pseudo degradation product DP6. EFO on direct hydrolysis at ester group forms DP3 and hydrolysis with esterification with co-solvent methanol forms pseudo degradation product DP5. Degradation of EFO in alkaline condition is shown in Figure 2a.

Photolytic condition - Under photolytic condition, EFO on dehydrogenation forms DP8 on which there is removal of benzyl group results in formation of DP7. EFO undergoes elimination of benzyl group forms DP9. EFO undergoes direct elimination of nitro phenyl group forms DP10. Degradation pathway of EFO in photolytic condition is shown in Figure $2 b$.

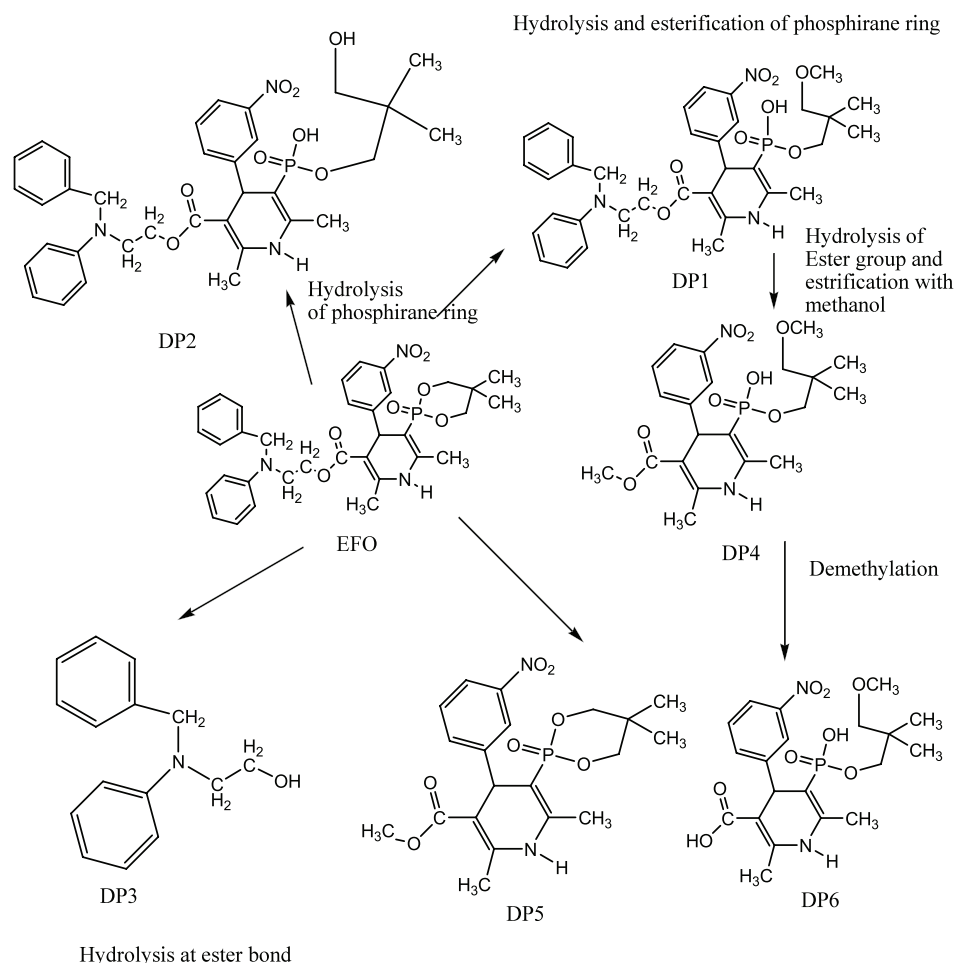

Hydrolysis of Ester group and estrification with methanol

Figure 2a. Degradation pathway of EFO in alkaline condition. 


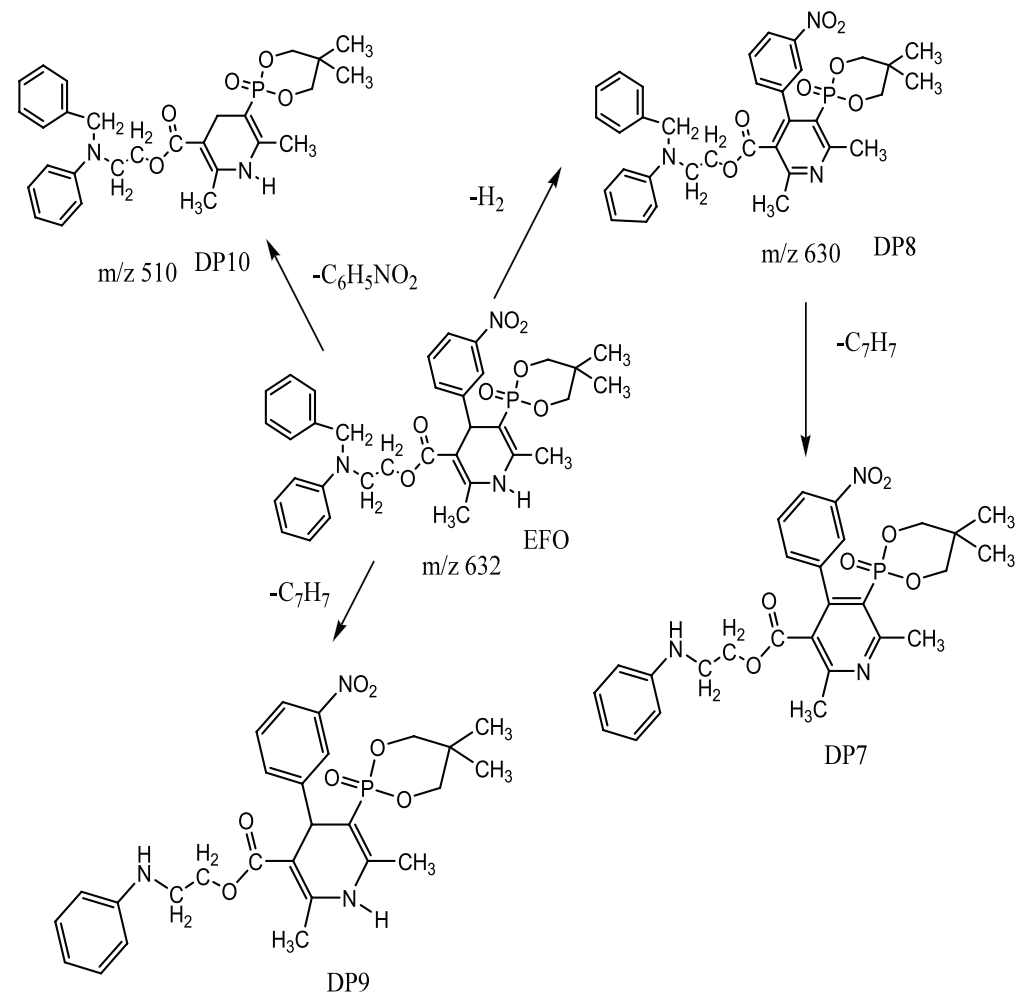

Figure 2b. Degradation pathway of EFO in photolytic condition.

\section{Structural interpretation of isolated degradation products}

Three degradation products such as DP1, DP3, and DP4 in alkaline conditions were isolated by preparative HPLC and subjected to NMR and IR analysis.

EFO - Structural analysis of EFO protonated mass at 632.2500 (calculated 632.2524). ${ }^{1} \mathrm{H}$ NMR spectra of EFO shows the presence of protons corresponding to methyl group at $0.87-0.86,0.93,2.20$, and 2.27-2.26 ppm. The presence of protons corresponding to methylene groups is indicated at 4.58 , $4.30-4.22$, and $4.05-3.99 \mathrm{ppm}$. The presence of protons in aromatic nitro group is indicated at 8.0, 7.9, 7.5, and $7.4 \mathrm{ppm}$. Protons of ethanolate are present in the region of 1.07-1.05, 3.47-3.42, and $6.72 \mathrm{ppm}$. IR spectra of EFO show the presence of secondary amino group at $3,435 \mathrm{~cm}^{-1}$ and the presence of aromatic groups at 3,185 and $3,083 \mathrm{~cm}^{-1}$. The presence of methyl and methylene groups is indicated at 2,967 and $2,820 \mathrm{~cm}^{-1}$. The presence of ester is indicated at $1,705 \mathrm{~cm}^{-1}$ and cyclic ether linkage at $1,645 \mathrm{~cm}^{-1}$ (Supple Table S1 and S5).

DP1 - Based on the analysis by LC-Q-TOF-MS, mass spectra of DP1 show protonated molecular ion peak at 664.2780 (calculated 664.2743), which is 32 amu more than that of EFO. This may be due to addition of methoxy group from co-solvent methanol. ${ }^{1} \mathrm{H}$ NMR spectra of DP1 were compared with that of EFO. In EFO, two methylene protons in phosphinane ring are indicated at 4.30 $4.22 \mathrm{ppm}$ and 4.05-3.99 ppm. In DP1, there is ring-opening of phosphinane ring, which is indicated by shifting of the methylene protons from 4.05-3.99 ppm to 3.34-3.31 ppm, and 3.55-3.51 $\mathrm{ppm}$. Addition of three protons from methyl group is indicated by chemical shift at $3.42-3.35 \mathrm{ppm}$ and the presence of one $-\mathrm{OH}$ group is indicated at 3.43-3.42 ppm. In ${ }^{13} \mathrm{CNMR}$ spectra of DP1, methylene groups in phosphinane ring are shifted from $74 \mathrm{ppm}$ to $69.89 \mathrm{ppm}$, and $66.41 \mathrm{ppm}$. Formation of additional methyl groups is indicated at $51.35 \mathrm{ppm}$. This indicates opening of phosphinane ring and esterification by methanol. I.R spectrum of DP1 indicates formation of broad peak covering hydroxyl group and -NH group. Broad peak is observed in the bending region covering 1,705, 1,598, and $1,775 \mathrm{~cm}^{-1}$. Peak at $1,645 \mathrm{~cm}^{-1}$ in EFO has been disappeared in DP1 formation of the peak at 1,598 and $1,574 \mathrm{~cm}^{-1}$ indicated ring-opening of phosphinane ring with formation of $\mathrm{P}-\mathrm{OH}$ group and esterification with methanol. Thus DP1 is characterized as 3-2-(N-benzylanilino) ethyl 3-oxo-2,2-dimethylpropyl hydrogen 1,4-dihydro-2,6-dimethyl-4-(3-nitrophenyl)pyridin-3-yl-3phosphonate (Supple Table S2 and S5) .

DP3 - Based on the analysis by LC-Q-TOF-MS, mass spectra of DP3 show protonated molecular ion peak at 228.1373 (Calculated 228.1344). DP3 is 404 amu less than EFO. In DP3, there is an absence of four methyl protons at $0.87-0.86,0.93,2.20$, and 2.27-2.26 and two methylene protons in phosphinane ring at $4.30-4.22$ and 4.05-3.99, and absence of aromatic nitro group at $7.9,8.0,7.4$, and $7.5 \mathrm{ppm}$. There is a formation of hydroxyl group which is indicated by a broad peak at $4.69 \mathrm{ppm}$. Protons at 15 and 16 position are shifted to upfield at 3.44-3.40 and 3.54-3.51 ppm. ${ }^{13}$ C NMR of DP3 spectra indicates the absence of methyl groups and methylene groups at $17.48,17.58$, and $18.59 \mathrm{ppm}$. It indicates the absence of ester group at $166.41 \mathrm{ppm}$. From this, it indicates that hydrolysis has been taken at ester functional group with the loss of aromatic nitro group, dihydropyridine ring, phosphinane ring, and formation of phenyl benzyl ethanol. I.R. spectra of DP3 show the presence of a broad peak at $3,304 \mathrm{~cm}^{-1}$. Aromatic ring is indicated by $3,000 \mathrm{~cm}^{-1}$ and methyl group is indicated at $2,970 \mathrm{~cm}^{-1}$. There is an absence of a peak at $1,705 \mathrm{~cm}^{-1}$. Aromatic $\mathrm{C}-\mathrm{H}$ bending is indicated by the appearance of a peak at $1,591 \mathrm{~cm}^{-1}$. Based on this, DP3 is characterized as 2-(N-benzyl-N-phenyl amino) ethanol (Supple Table S3 and S5). 
DP4 - Based on the analysis by LC-Q-TOF-MS, mass spectra of DP4 show protonated molecular ion peak at 469.1705 (Calculated 469.17). In DP4, there is an absence of two rings, which are indicated by the absence of peaks in the region from 6.3 to $7.14 \mathrm{ppm}$ and absence of two methylene protons, which are indicated by the absence of a peak at 3.59 and $3.62 \mathrm{ppm}$. Formation of two methyl groups is indicated at 4.58, 4.30-4.22 ppm and there is a formation of one hydroxyl group at $3.33-3.28 \mathrm{ppm}$. One of the methylene groups is shifted to $3.63-3.51 \mathrm{ppm} .{ }^{13} \mathrm{C}$ NMR spectra of DP4 indicate the absence of aromatic rings and the absence of two methylene groups at 53 and $60 \mathrm{ppm}$. Esterification with methanol and formation of additional methyl group is indicated at 51.20 and $51.15 \mathrm{ppm}$. DP4 might have been formed from DP1 by hydrolysis at ester linkage with the loss of two aromatic rings and esterification with co-solvent methanol. IR spectra of DP4 indicate broad peak covering-OH group and -NH group. The presence of aromatic ring is indicated in the region of 3,192 and 3,166 $\mathrm{cm}^{-1}$. Methyl groups are indicated in the region of 2,951, 2,873, and 2,848. Opening of phosphinane ring and further etherification by methanol is shown by ether linkage at $1,645 \mathrm{~cm}^{-1}$. Based on the above analysis, DP4 is characterized as 3-methoxy-2,2-dimethylpropyl hydrogen 1,4-dihydro-2,6-dimethyl-5-methyloxycarbonyl-4-(3-nitro) phenylpyridin-3-yl-3-phosphonate (Supple Table S4 and S5).

\section{Mechanism of formation of degradation products DP1, DP3,} and DP4

DP1 - In alkaline condition, ring-opening of phosphinane takes place. Methoxide ion acts as nucleophile and it attacks on carbocation of phosphinane ring and forms the intermediate. Abstraction of a proton by negatively charged oxygen in the intermediate results in formation of DP1. Mechanism of formation of DP1 is shown in Figure 3 a.

DP3 - EFO possess ester functional group. Nucleophilic attack of hydroxide ion takes place on ester. Cleavage of carbonyl bond takes place and tetrahedral intermediate is formed. Cleavage takes place at tetrahedral intermediate, there is a formation of 2-(N-benzyl-N-phenylamino) ethoxide ion. Abstraction of proton by 2-(N-benzyl-N-phenylamino) ethoxide ion results in formation of corresponding acid and DP3. Mechanism of formation of DP3 is shown in Figure 3b.

DP4 - DP4 is formed from DP1 by alkaline hydrolysis. In DP1, there nucleophilic attack of methoxide ion to carbonyl carbon and there is formation of tetrahedral intermediate. With further loss of benzyl phenyl ethyl amino group, there is a formation of DP4. Mechanism of formation of DP4 is shown in Figure 3c.

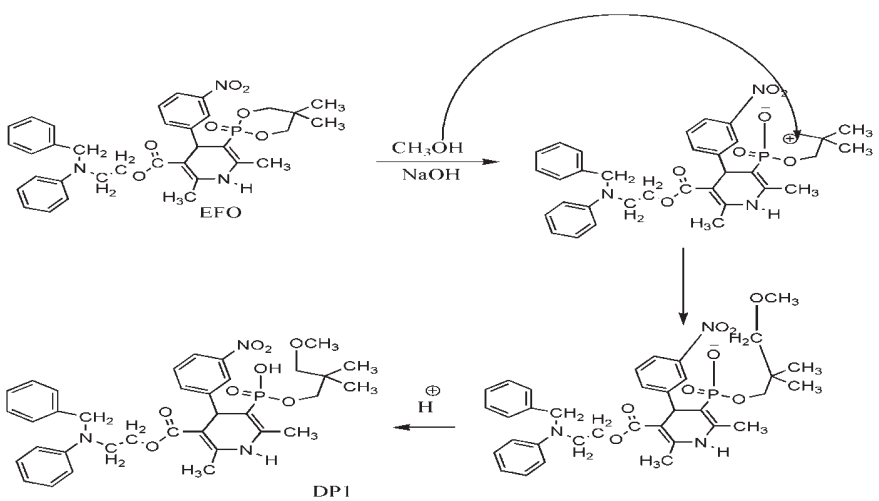

Figure 3a. Mechanism of formation of DP1.

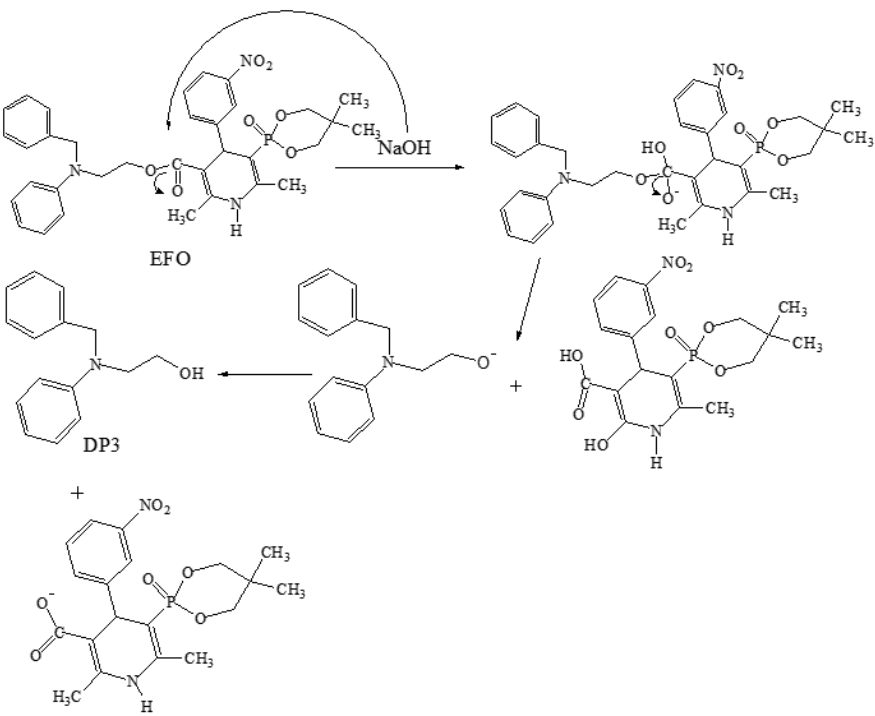

Figure 3b. Mechanism of formation of DP3.
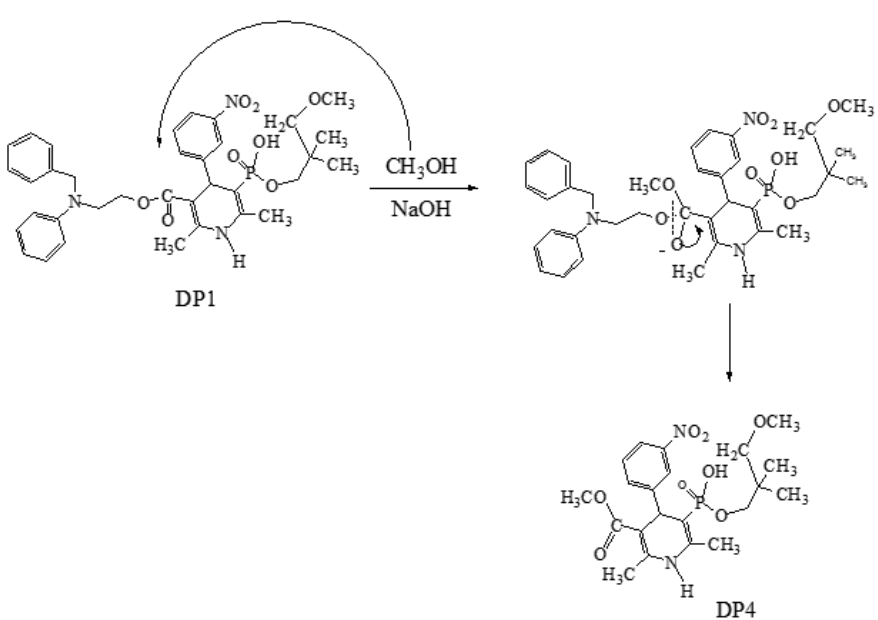

Figure 3c. Mechanism of formation of DP4.

\section{CONCLUSION}

Forced degradation study was performed for analysis of EFO. Degradation was observed in alkaline and photolytic conditions, and degradation products were identified by LC-ESI-QTOF-MS. Three degradation products in alkaline conditions were isolated by preparative HPLC and characterized by NMR and I.R. techniques. DP1 is characterized as 3-2-(N-benzylanilino)ethyl 3-oxo-2,2-dimethylpropyl hydrogen 1,4-dihydro-2,6-dimethyl-4(3-nitrophenyl)pyridin-3-yl-3-phosphonate, DP3 is characterized as 2-(N-benzyl-N-phenylamino)ethanol, and DP4 is characterized as 3-methoxy-2,2-dimethylpropyl hydrogen 1,4-dihydro-2,6dimethyl-5-methyloxycarbonyl-4-(3-nitro)phenylpyridin-3-yl3 -phosphonate. Degradation pathways in alkaline and photolytic conditions were proposed.

\section{ACKNOWLEDGMENT}

Charu Pandya is highly thankful to University Grants Commission, New Delhi and Government of India for availing Senior Research Fellowship [letterno.F.No. 25-1/2014-15(BSR)/7129/2007(BSR)]. 


\section{DECLARATION OF INTEREST}

The authors declare that there is no conflicts regarding publication of this paper.

\section{ABBREVIATIONS}

$\begin{array}{ll}\text { ACN } & \text { Acetonitrile } \\ \text { BDS } & \text { Base Deactivated Silica } \\ \text { DP } & \text { Degradation Product } \\ \text { DMSO } & \text { Dimethyl sulphoxide } \\ \text { FDA } & \text { Food and Drug Administration } \\ \text { HPLC } & \text { High Performance Liquid Chromato- } \\ & \text { graphy } \\ \text { ICH } & \text { International Conference on Harmoni- } \\ & \text { zation } \\ \text { IR } & \text { Infra Red } \\ \text { LC-Q-TOF-MS } & \text { Liquid Chromatography Quadrupole } \\ & \text { Time of Flight Mass Spectrometer } \\ \text { LOD } & \text { Limit of Detection } \\ \text { LOQ } & \text { Limit of Quantification } \\ \text { NMR } & \text { Nuclear Magnetic Resonance } \\ \text { PDA } & \text { Photo Diode Array } \\ \text { RT } & \text { Room Temperature } \\ \text { RSD } & \text { Relative Standard Deviation }\end{array}$

\section{REFERENCES} drugs/DB0923

Drugbank.ca. 2019. Available via https://www.drugbank.ca/

Food and Drug Administration (FDA). Guidance for industry: analytical procedures and methods validation. pp. 4-12, 2000.

Hikaru T, Koki S. Efonidipine hydrochloride: a dual blocker of L-and T-type $\mathrm{Ca}(2+)$ channels. Cardiovas Drug Rev 2002; 20:81-92.

International Conference on Harmonization (ICH). Guidance for industry: Q2(R1) validation of analytical procedures: text and methodology. pp. 6-13, 2005.

International Conference on Harmonization (ICH). Q1A(R2) stability testing of new drug substances and products. pp. 5-16, 2003.

International Conference on Harmonization (ICH). Q1B stability testing: photostability testing of new drug substances and products. pp. 4-6, 1996.

Kumar A, Shoni SK, Dahiya M, Kumar R, Yadav AK, Kumar V, Choudhary H. Development and validation of liquid chromatography(RPHPLC) methodology for estimation of Efonidipine $\mathrm{HCl}$ Ethanolate. Pharm Anal Acta 2017; 8:1-6.
Liu M, Deng M, Zhang D, Wang X, Ma J, Zhao H, Zhang L, Tong Y, Liu H. A chiral LC-MS/MS method for the stereo specific determination of efonidipine in human plasma. J Biomed Pharm Anal 2016; 122:35-41.

Liu M, Zhao H, Tong Y, Zhang D, Wang X, Zhang L, Han J, Liu H. 2015. Determination of efonidipine in human plasma by LC-MS/MS for pharmacokinetic applications. J Pharm Biomed Anal 2015; 103:1-6.

Masuda Y, Tanaka H. Efonidipine hydrochloride: a new calcium channel antagonist. Cardiovas. Drugs Rev 1994; 12:123-35.

Nakano N, Ishimitsu T, Takahashi T, Inada H, Okamura A, Ohba S, Matsuoka H. Effects of efonidipine, an L-and T-type calcium channel blocker, on the rennin-angiotension -aldosterone system in chronic hemodialysis patients. Inter Heart J 2010; 51:188-92.

National Center for Biotechnology Information. Pubchem Database. CID $=163838$. Available via https://pubchem.ncbi.nlm.nih.gov/ compound/Efonidipine-hydrochloride-ethanolate (Accessed 4 December 2018).

Otsuka M, Maeno Y, Fukami T, Inoue M, Tagami T, Ozeki T. Development considerations for ethanolates with regard to stability and physicochemical characterization of efonidipine hydrochloride ethanolate. Cryst Eng Comm 2015; 17:743

Otsuka M, Maeno Y, Fukami T, Inoue M, Tagami T, Ozeki T. Solid dispersions of efonidipine hydrochloride ethanolate with improved physicochemical and pharmacokinetic properties prepared with microwave treatment. Euro. J Pharmaceu Biopharmaceu 2016; 108:25-31.

Singh S, Bakshi M. Guidance on conduct of stress tests to determine inherent stability of drugs. Pharmaceu Tech On-Line 2000; 24:1-14.

Singh S, Handa T, Narayanam M, Sahu A, Junwal M, Shah RP. A critical review on the use of modern sophisticated hyphenated tools in the characterization of impurities and degradation products. J Pharm Biomed Anal 2012; 69:148-73.

Wang N, Ye L, Zhaon BQ, Yu JX. Spectroscopic studies on interaction of efonidipine with bovine serum albumin. Brazilian J Med Bio Res 2008; 41:589-95.

How to cite this article:

Pandya CP, Rajput SJ. Forced degradation study of efonidipine $\mathrm{HCl}$ ethanolate, characterization of degradation products by LC-Q-TOF-MS and NMR. J Appl Pharm Sci, 2020; 10(04):075-099. 


\section{SUPPLEMENTARY DATA}

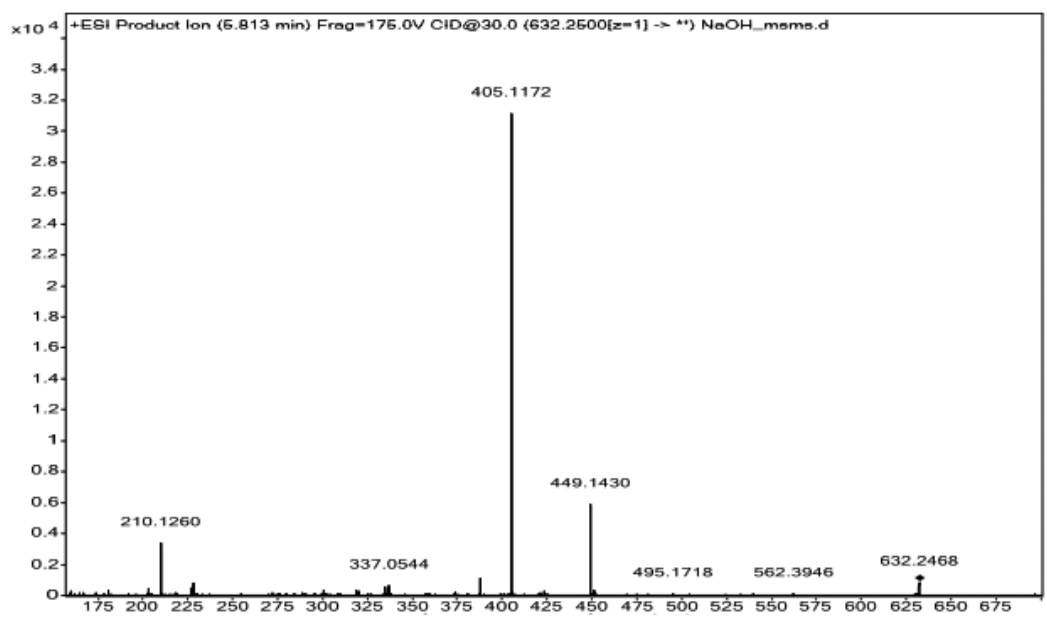

Figure. S1a . ESI-MS/MS spectra of EFO.

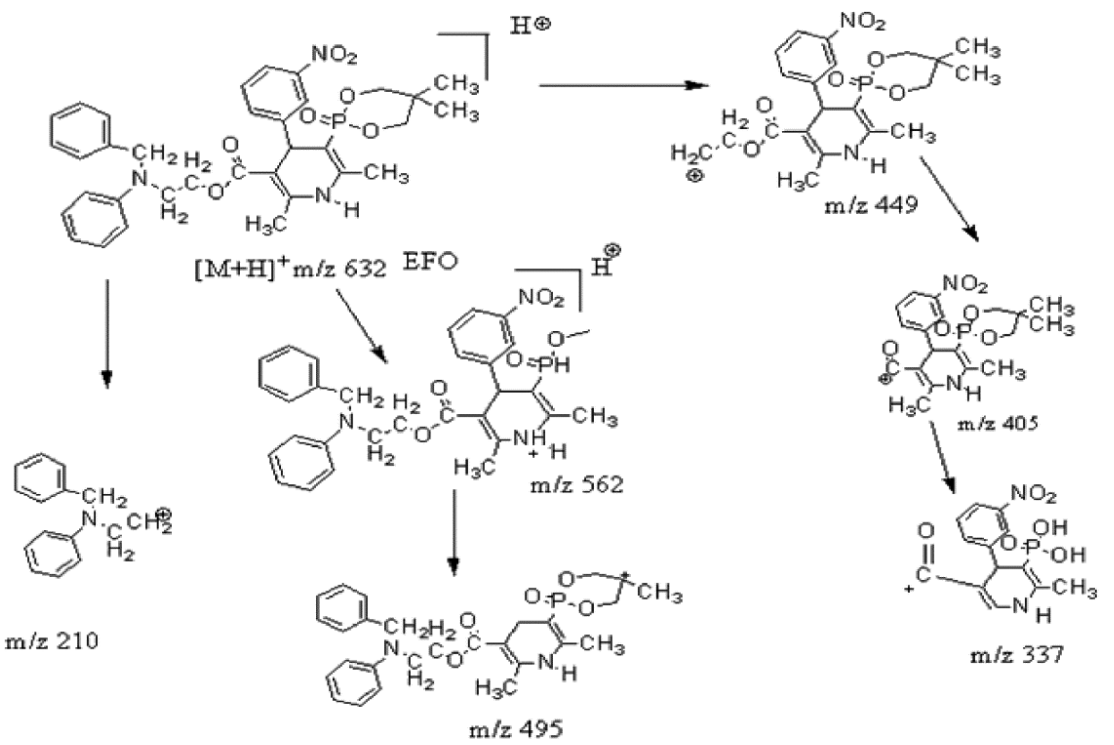

Figure. S1b. Fragmentation pathway of EFO.

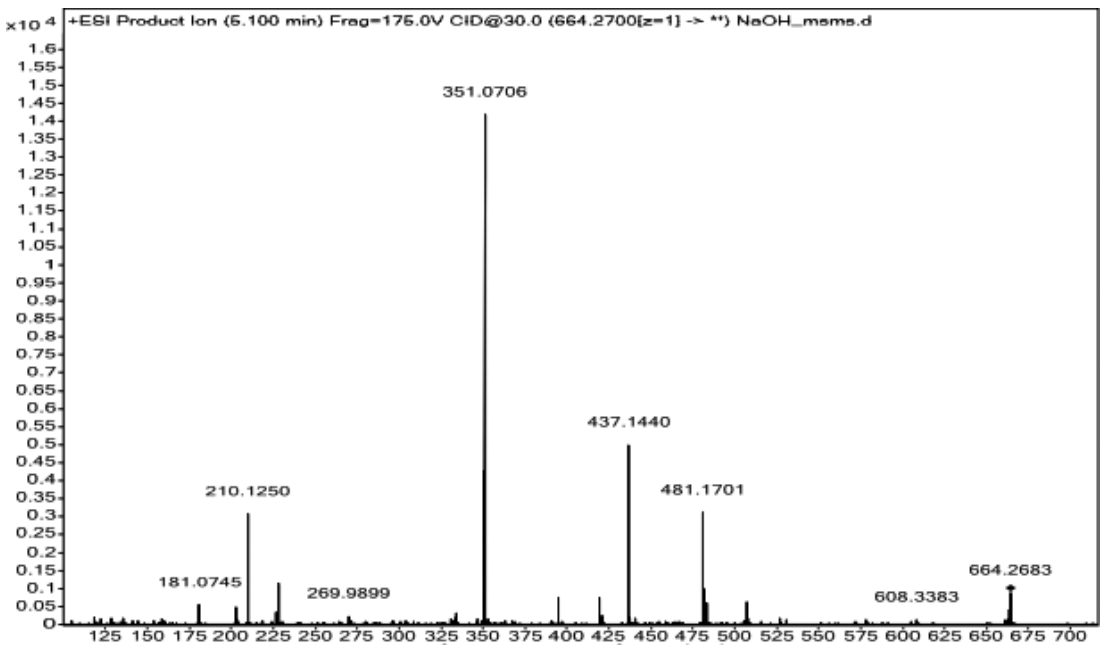

Figure. S2 -a. ESI-MS/MS spectra of DP1. 


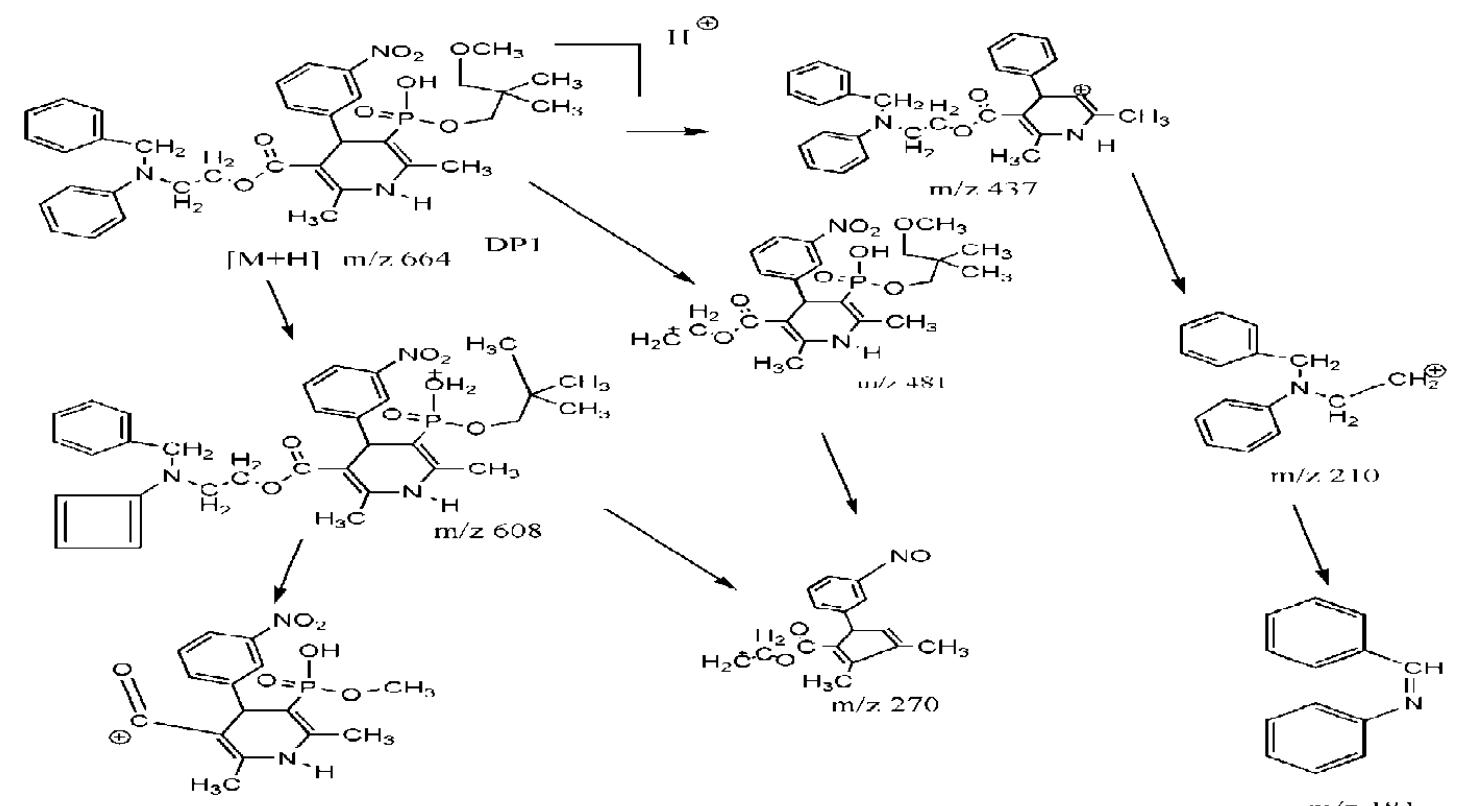

$\mathrm{m} / \mathrm{z} 351$

Figure. S2 b. Fragmentation pathway of DP1.

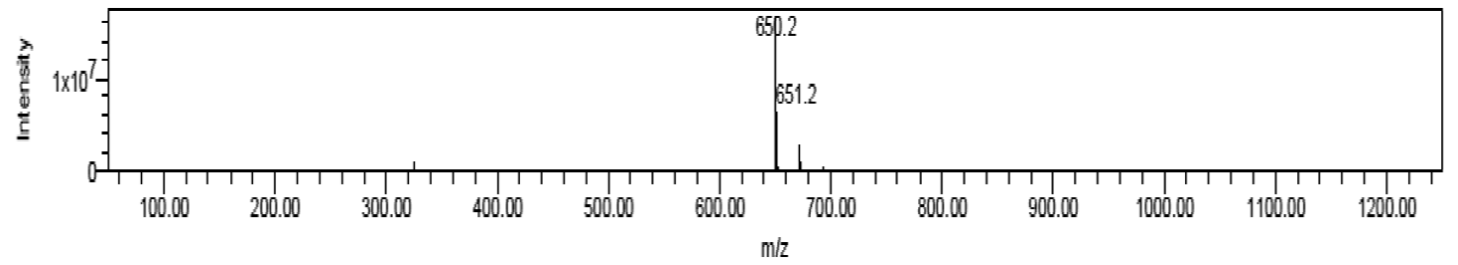

Figure. S3 a. ESI-MS spectra of DP2.

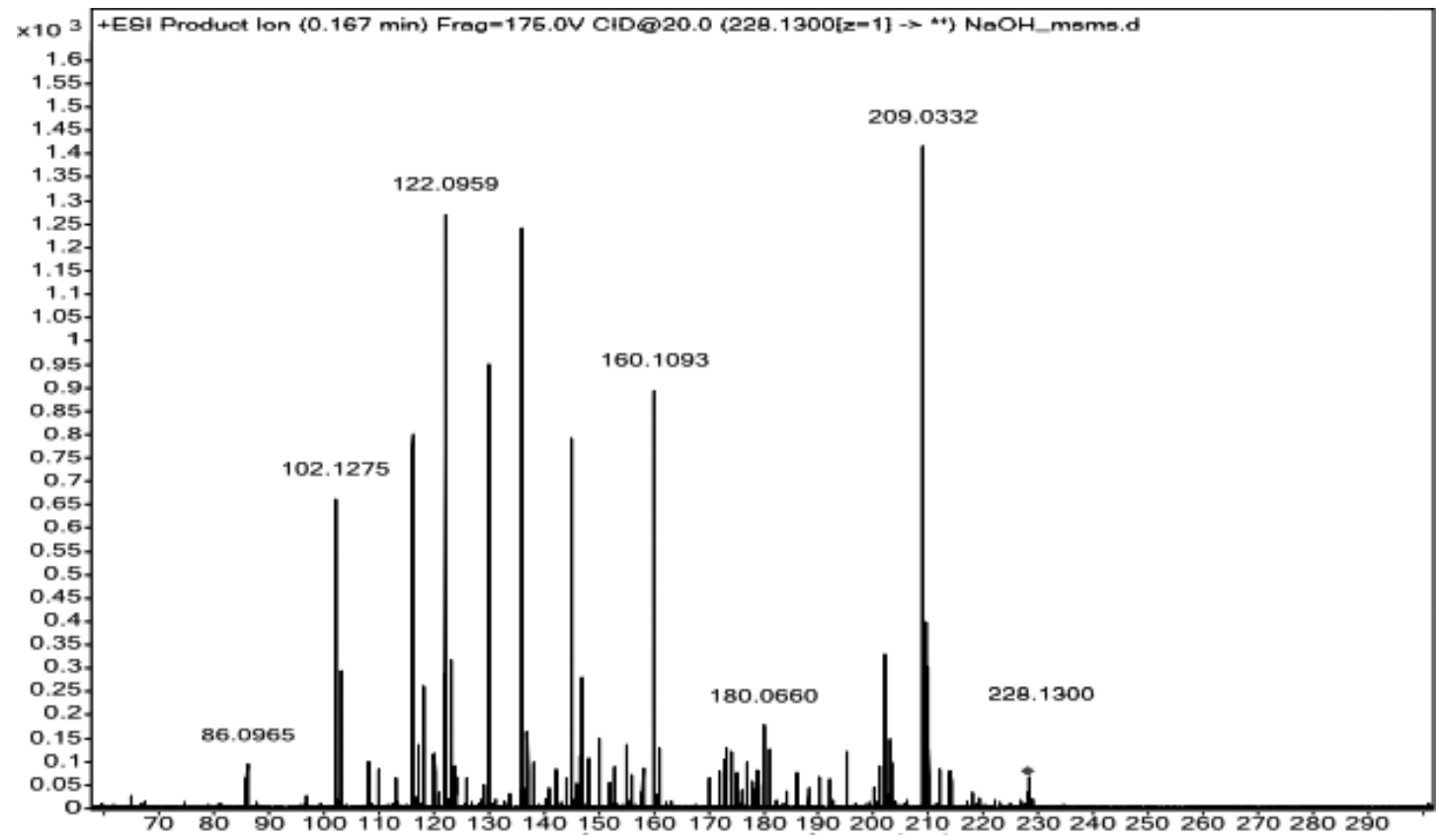

Figure. S4 a. ESI-MS/MS spectra of DP3 


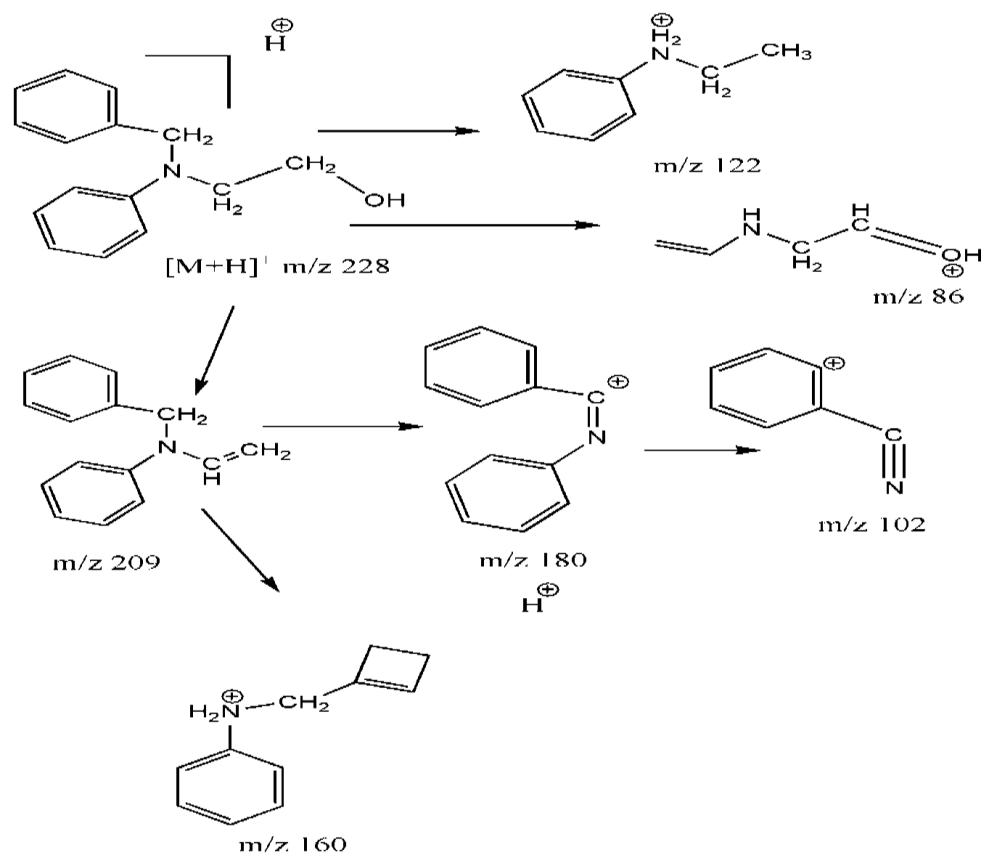

Figure. S4 b. Fragmentation pathway of DP3.

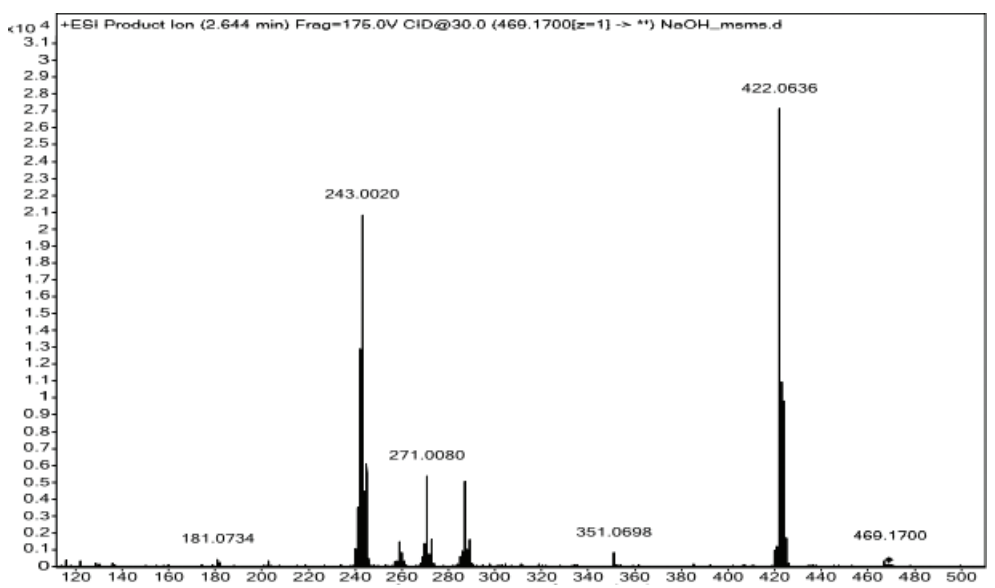

Figure. S5 a . ESI-MS/MS spectra of DP4.

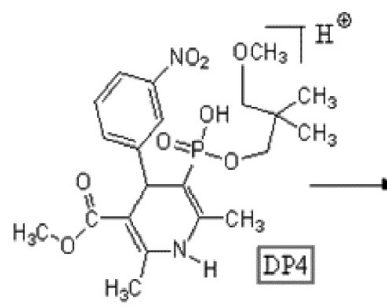

$[\mathrm{M}+\mathrm{H}]^{+} \mathrm{m} / \mathrm{z} 469.17$

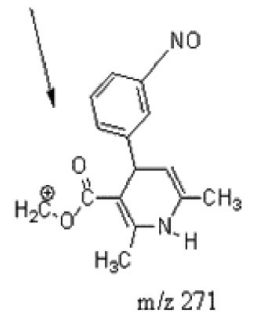<smiles>COC(=O)C1=C(C)NC(C)=C(P(=O)(OCC(=O)C2=C(C)NC(C)=C(P(=O)(O)OC)C2c2cccc([N+](=O)[O-])c2)OCC(C)(C)C)C1c1cccc([N+](=O)[O-])c1</smiles>

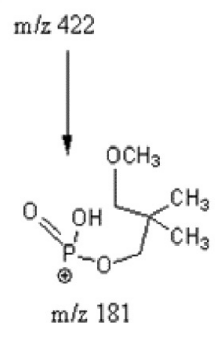

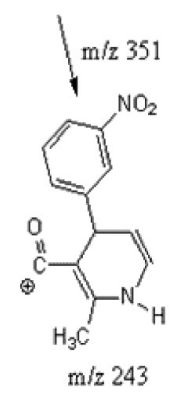

Figure. S5 b . Fragmentation pathway of DP4. 


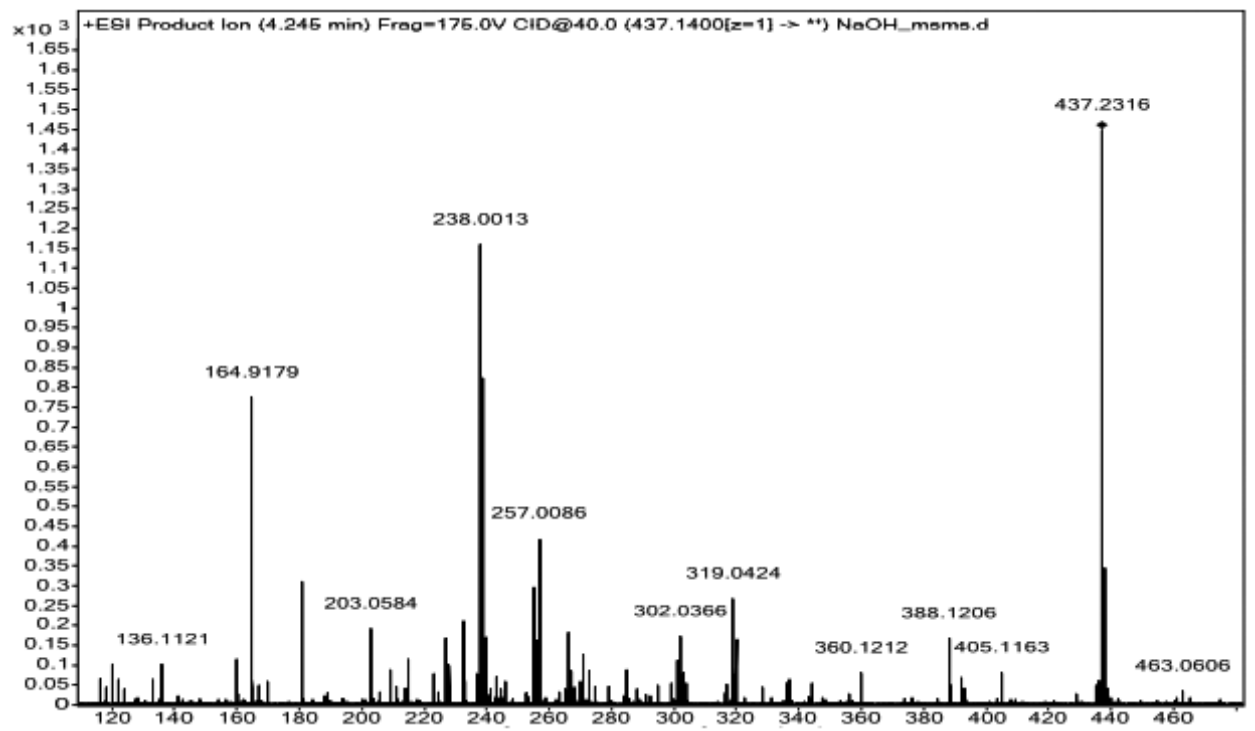

Figure. S6 a. ESI-MS/MS spectra of DP5.

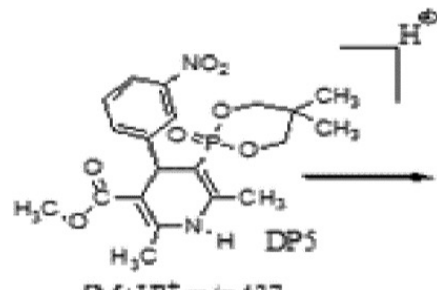

$[\mathrm{M}+\mathrm{H}]^{+} \mathrm{m} / \mathrm{z} 437$
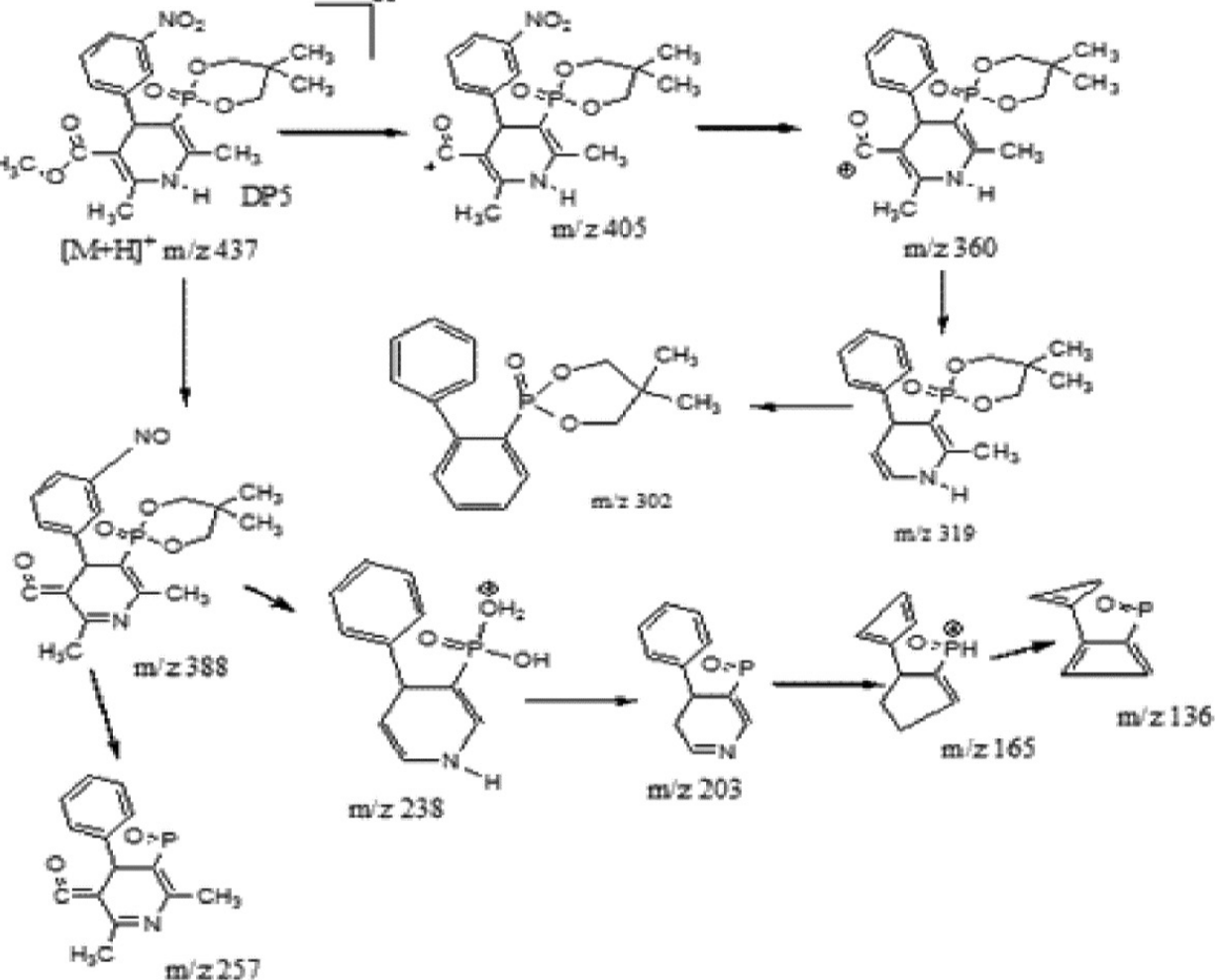

Figure. S6 b . Fragmentation pathway of DP5.

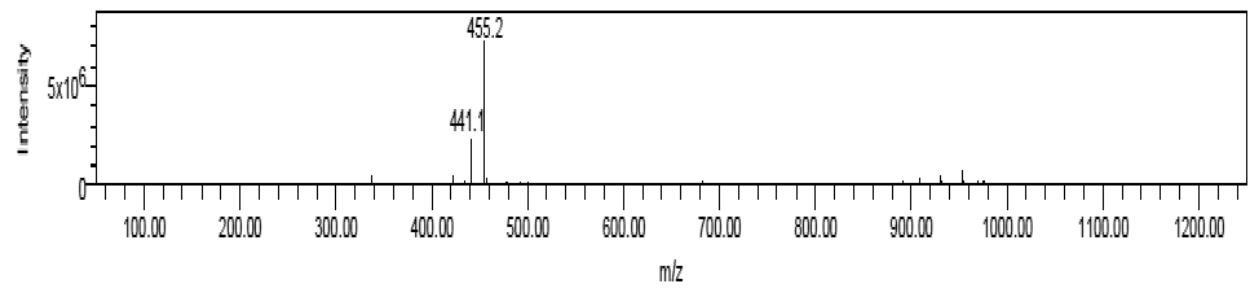

Figure.S7 a. ESI-MS spectra of DP6. 


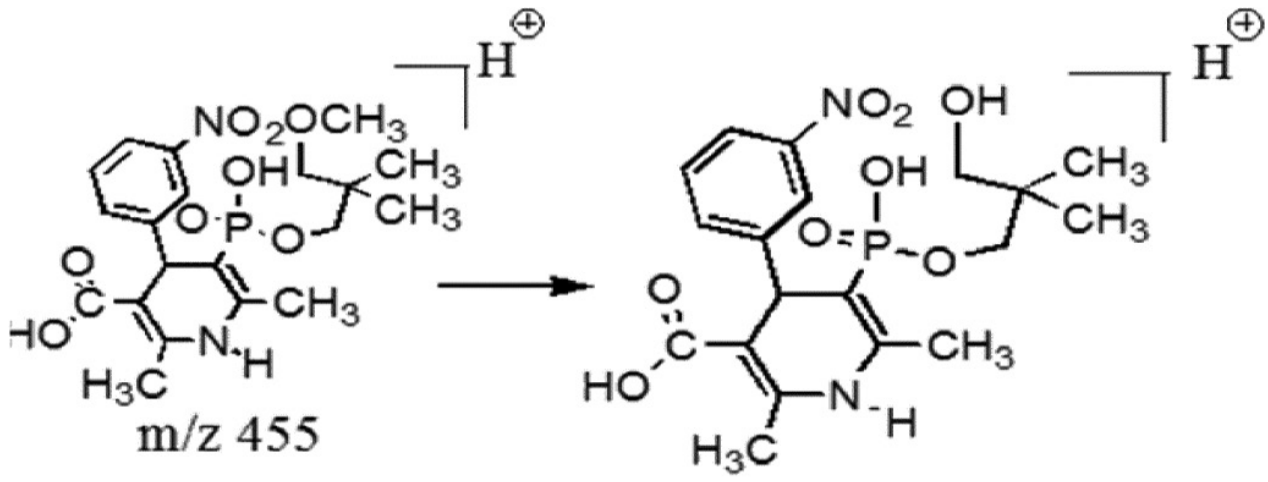

$\mathrm{m} / \mathrm{z} 441$

Figure.S7 b. Fragmentation pathway of DP6.

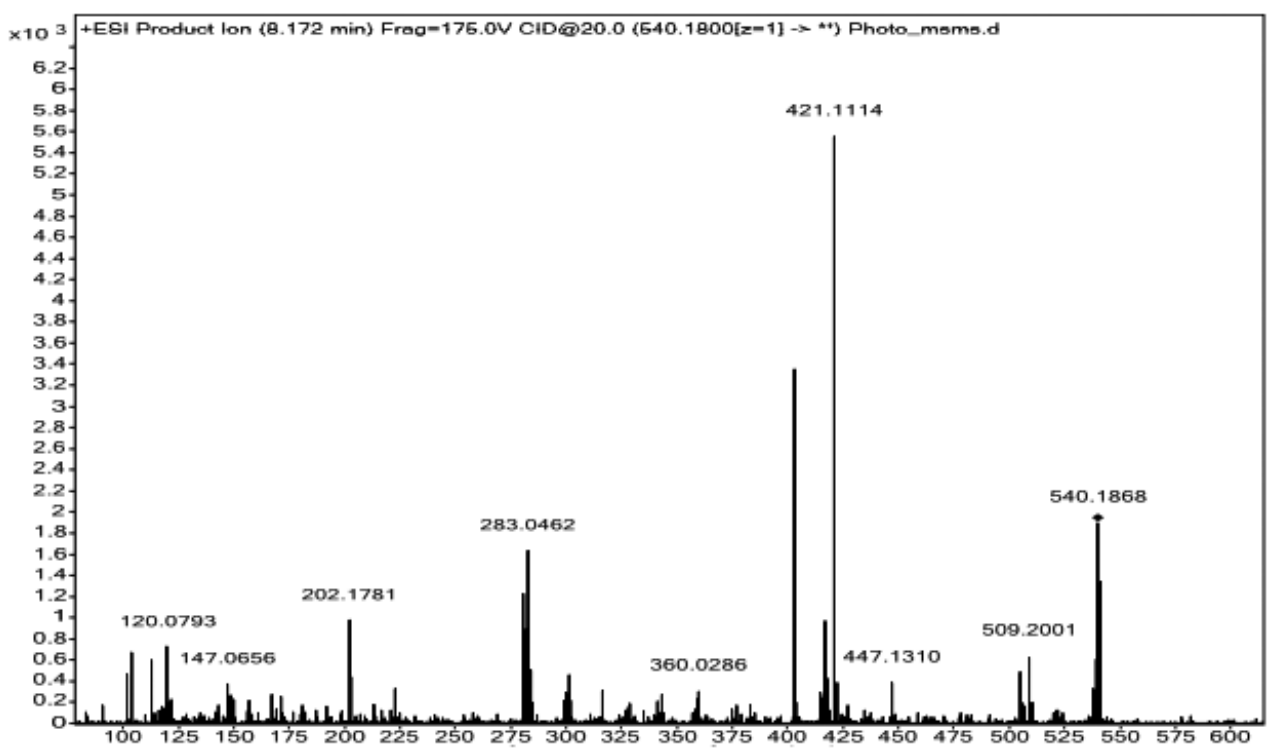

Figure. S8 a. ESI-MS/MS spectra of DP7.

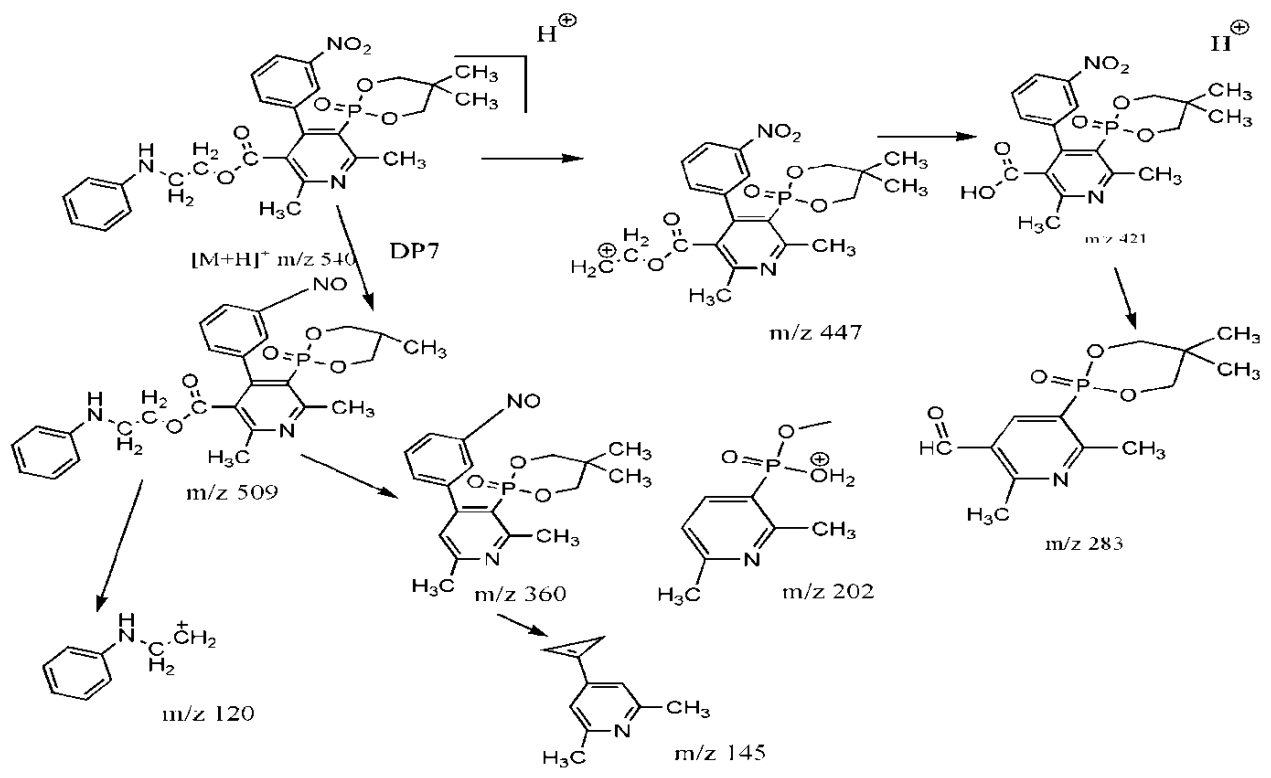

Figure. S8 b. Fragmentation pathway of DP7. 


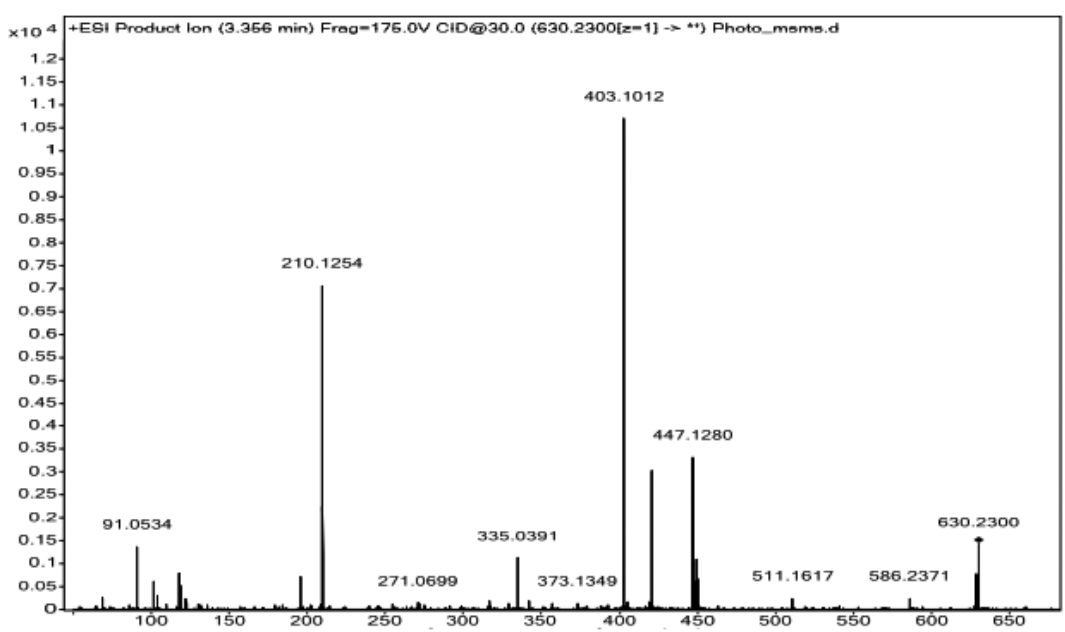

Figure. S9 a. ESI-MS/MS spectra of DP8.

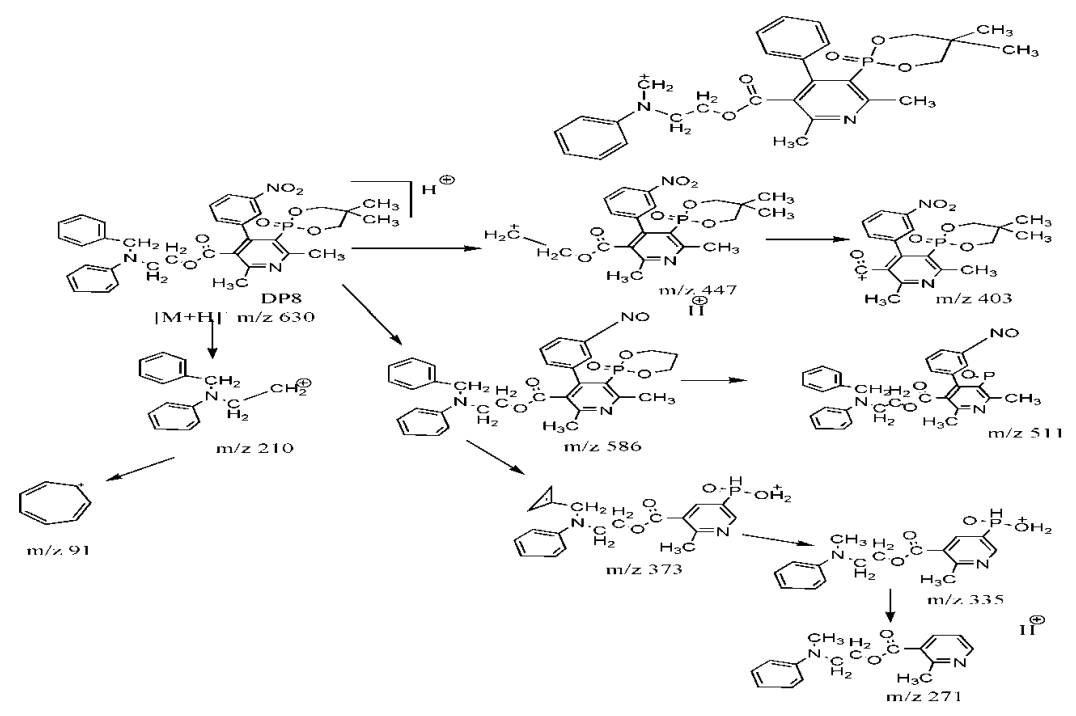

Figure. S9 b. Fragmentation pathway of DP8.

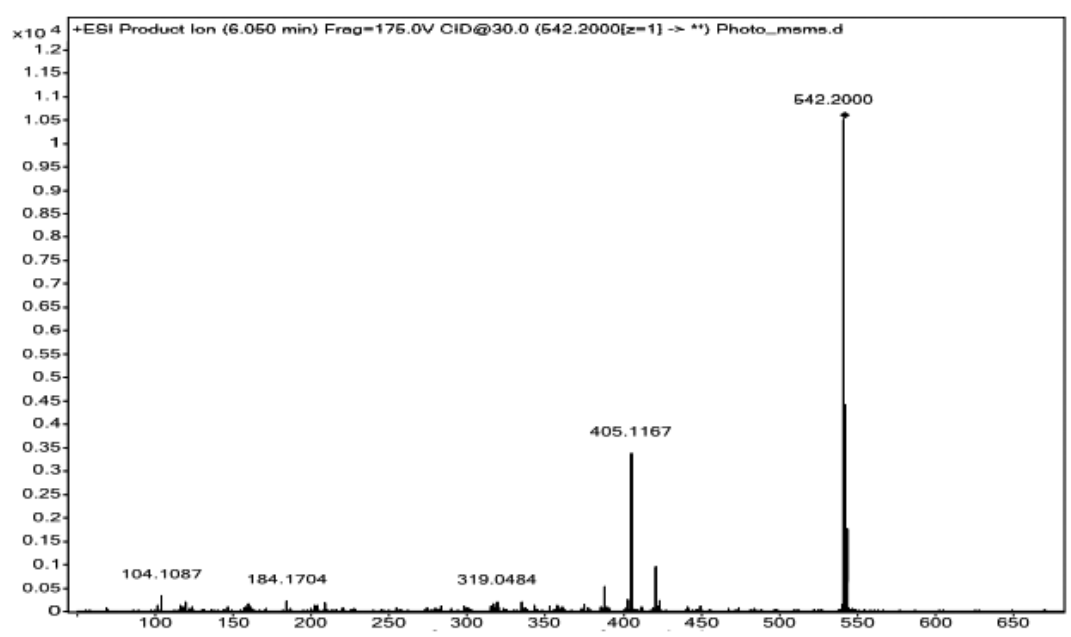

Figure. S10 a. ESI-MS/MS spectra of DP9. 

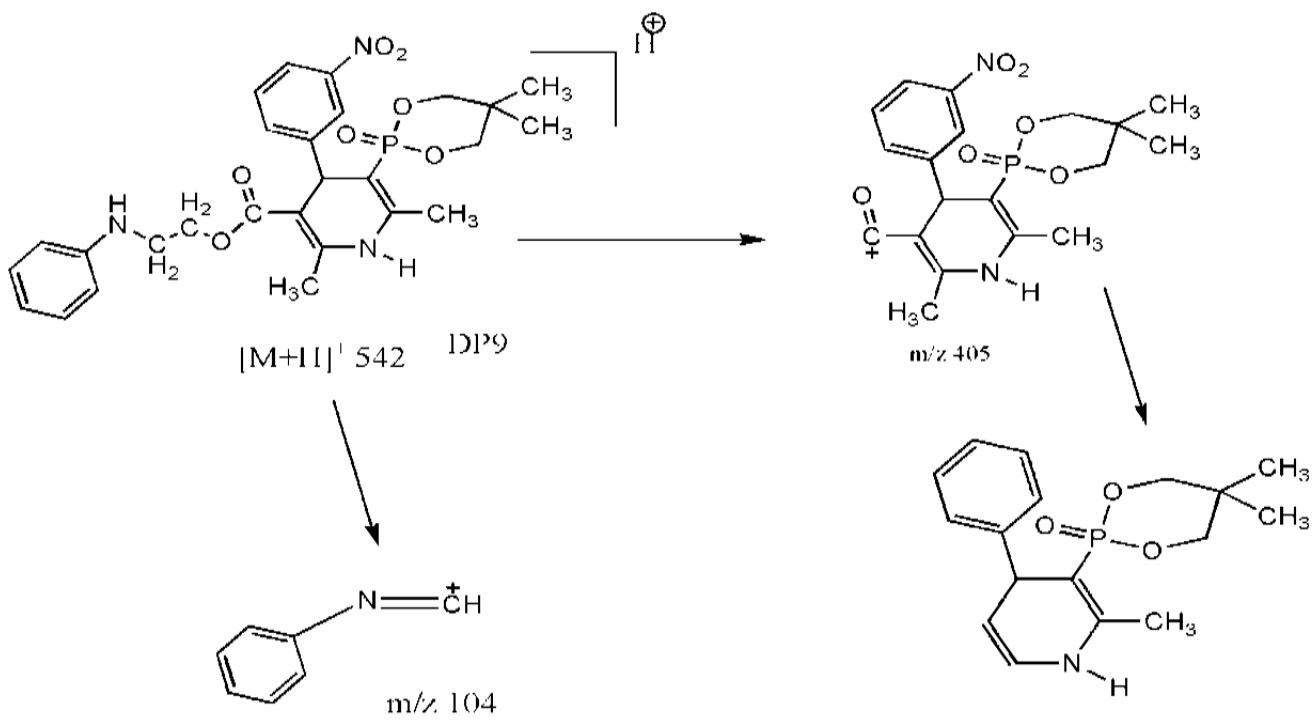

$\mathrm{m} / \mathrm{s} .319$

Figure. S10 b. Fragmentation pathway of DP9.

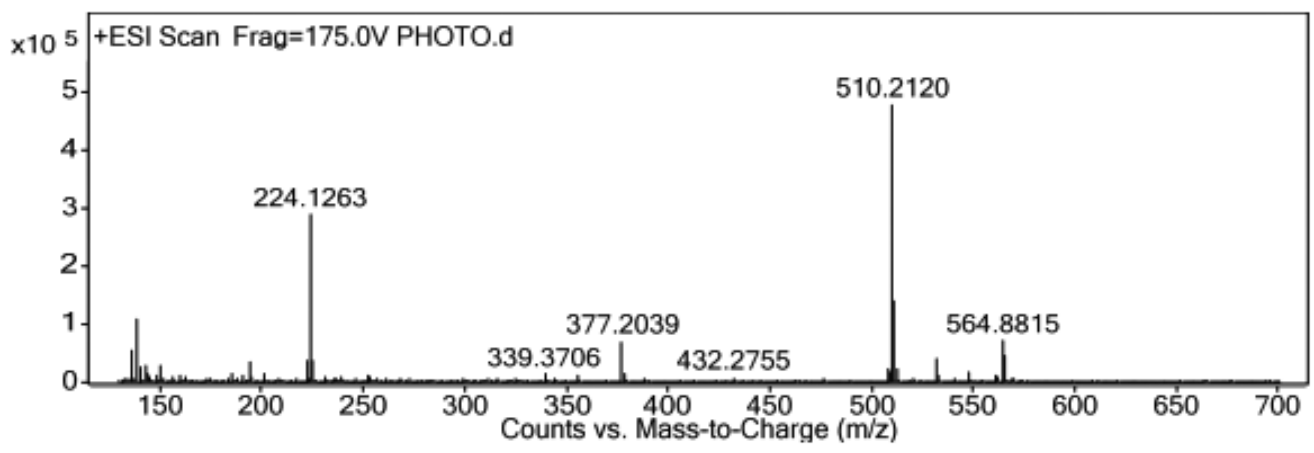

Figure. S11 a. ESI-MS spectra of DP10.

EFO

BRUKER
AVANCE II 400 NMR

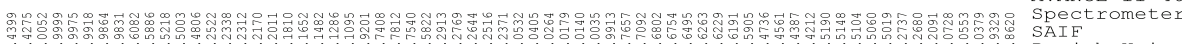

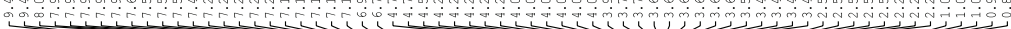

Panjo university

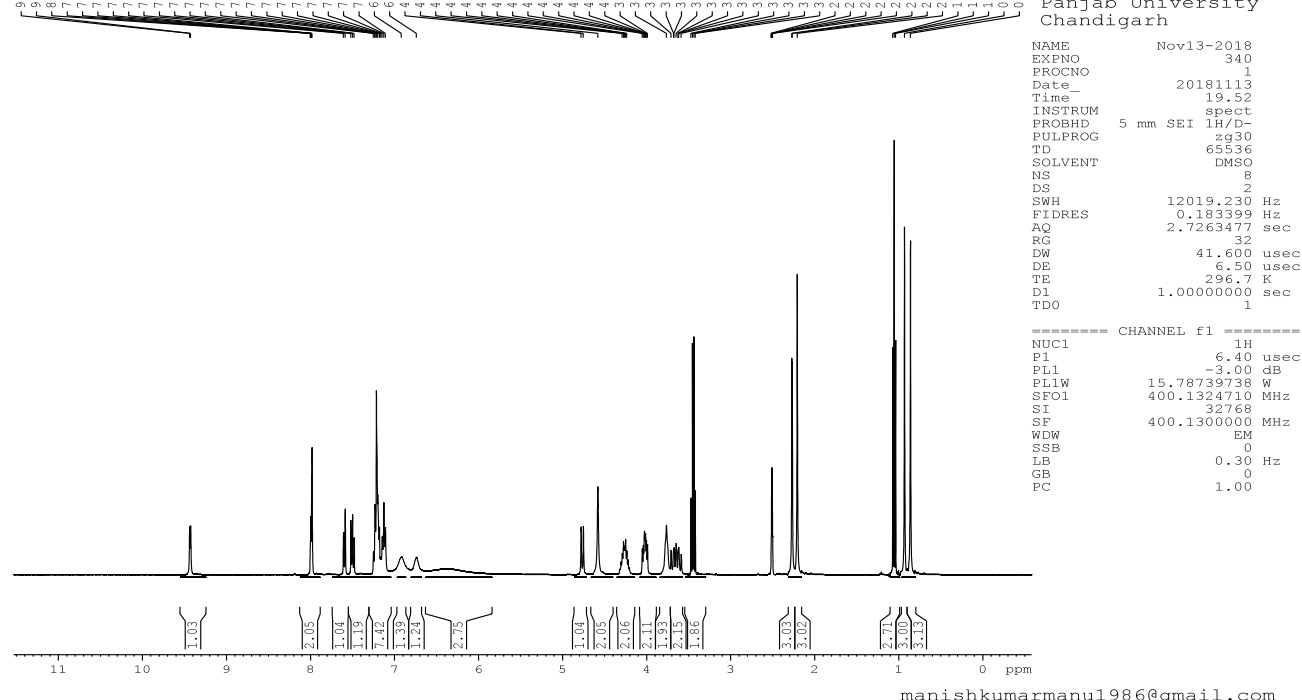

Figure. S12 a.${ }^{1} \mathrm{H}$ NMR spectra of EFO. 
EFO

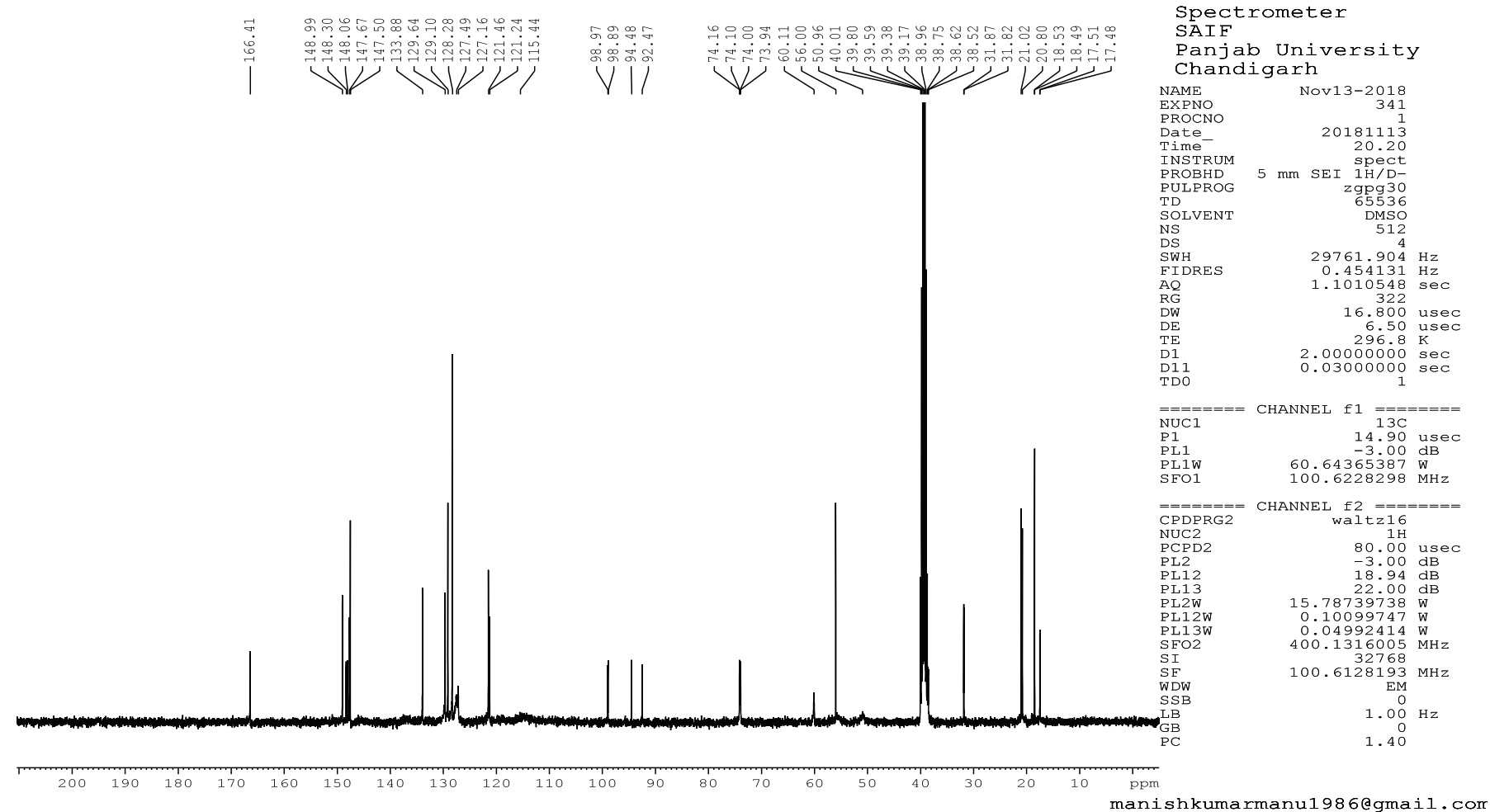

Figure. S12 b $\cdot{ }^{13} \mathrm{C}$ NMR spectra of EFO.

EFO

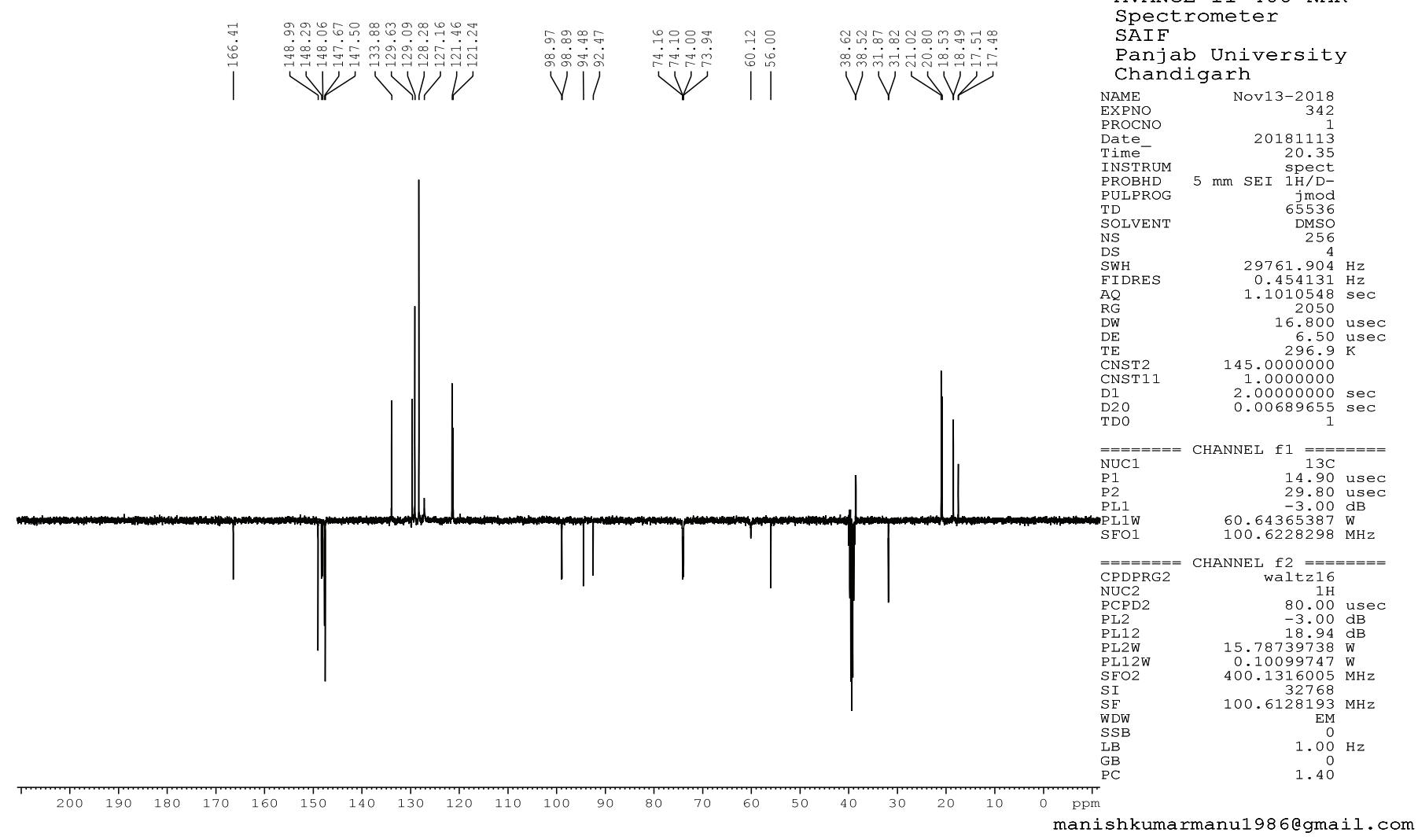

Figure. S12 c. APT spectra of EFO. 
EFO

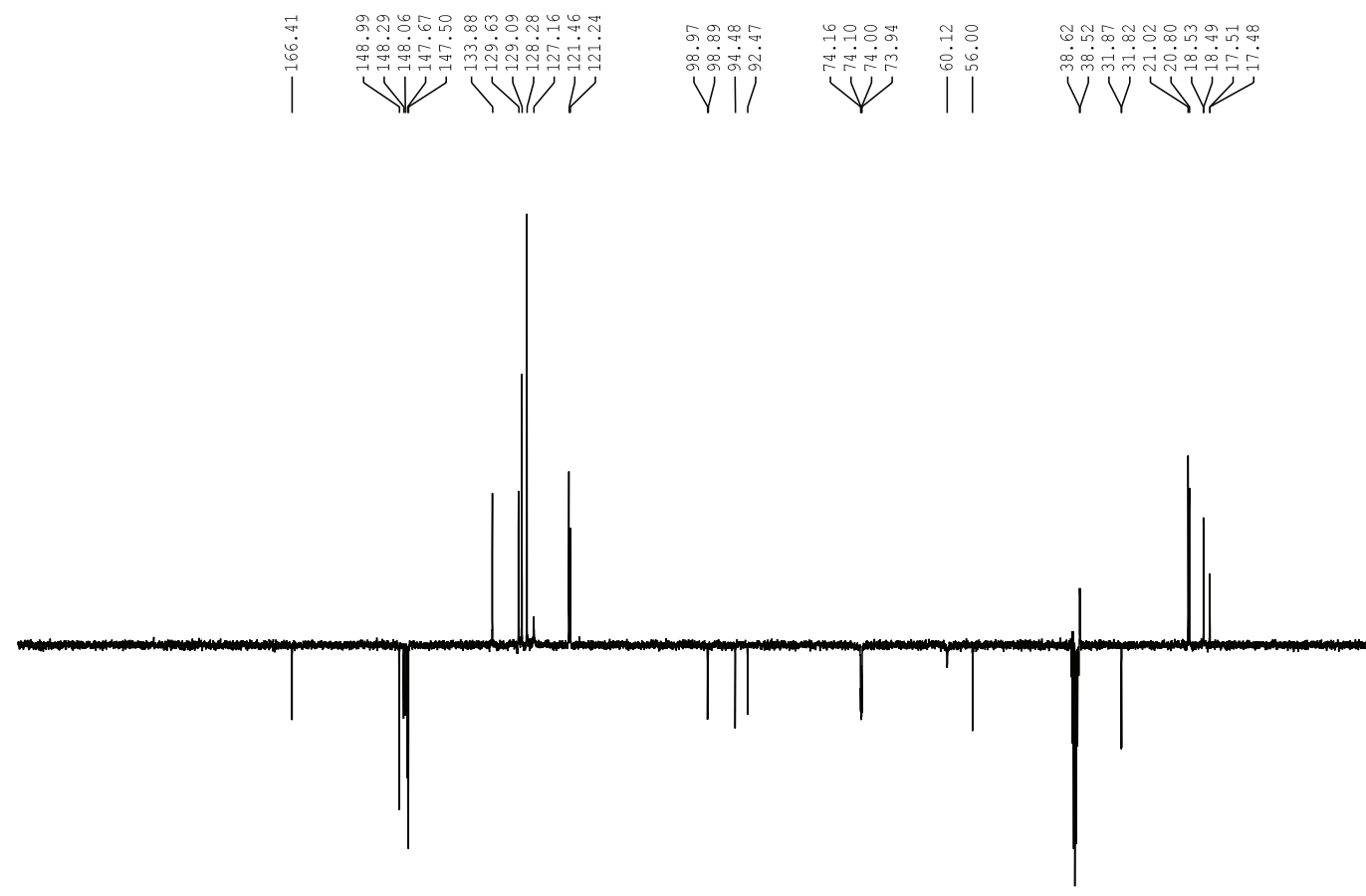

BRUKER

AVANCE II 400 NMR

Spectrometer

SAIF

Panjab University

Chandigarh

NAME

EXPNO Nov13-2018

$\begin{array}{ll}\text { PROCNO } & 1 \\ \text { Date- } & 2018111^{1}\end{array}$

PROBHD $5 \mathrm{~mm}$ SEI $\begin{gathered}\text { spect } \\ 1 \mathrm{H} / \mathrm{D}-\end{gathered}$

PULPROG 5 MUT SEI 1 jmod

TD

NS

FID

AQ

DW
DE
TE

CNST2 2

D2 0
TDO

$=======$ CHANNEL fl $========$

P1
PL1

PLIW

SFO1

CPDPRG2

$\mathrm{PL} 2$
$\mathrm{PL} 12$

PL2W

SFO2
SI
SE
WDW

WDW

LB

PC

$9761.904 \mathrm{~Hz}$

i010548 sec

6.50 usec

$.00000000 \mathrm{se}$

$.00689655 \mathrm{sec}$

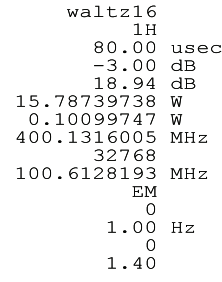

Figure. S13 a . ${ }^{1} \mathrm{H}$ NMR spectra of DP1.

EFO DP1
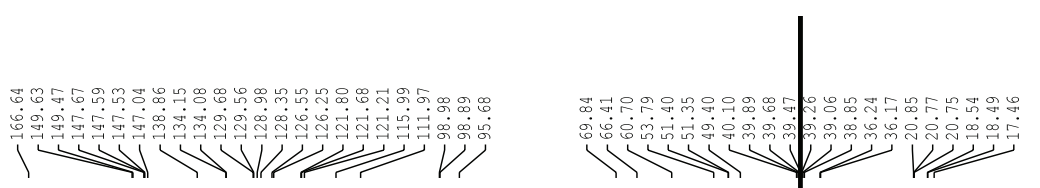

BRUKER

AVANCE II 400 NMR

Spectrometer

SAIE

Panjab University

Chandigarh

NAME
EXPNO
PROCNO

Date-

$5 \mathrm{~mm} \mathrm{spect}$

PROBHD

TD
SOLVENT

NS

DS
SWH
FIDRES
AQ

AQ
RG
DW

DW

DE
TE
D1
D11

zgpg 30
65536 
EFO DP1

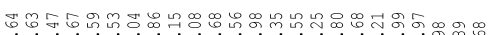

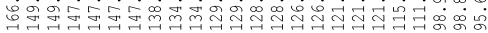

$\longrightarrow$

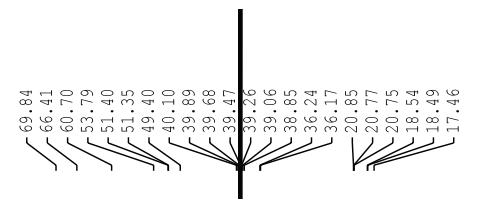

BRUKER

AVANCE II 400 NMR Spectrometer

SAIF

Panjab University Chandigarh

NAME

NAME
EXXNO
PROCNO

PROCNO
Date-
Time

PROBHM spect

PULPROG 5 mm SEI $\begin{array}{r}\text { ZgPg } 30 \\ 65536\end{array}$

TD
SOLVENT
NS

NS

SWH

AQ
RG

RG
DW
DE

$\mathrm{DE}$
$\mathrm{TE}$
$\mathrm{D} 1$
$\mathrm{D} 11$

TDO

$====-1$

NUC1
P1 1
PL1
PL1W

SFO1

$=======$
CPDPRG2

CPDPRC2
NUC2
PCPD2

PCPD2

$\mathrm{PL} 12$

$\mathrm{PL} 13$
$\mathrm{PL} 2 \mathrm{~W}$
$\mathrm{PL} 12 \mathrm{~W}$

PL12W
PL13W

SI

WDW

$G B$
PC

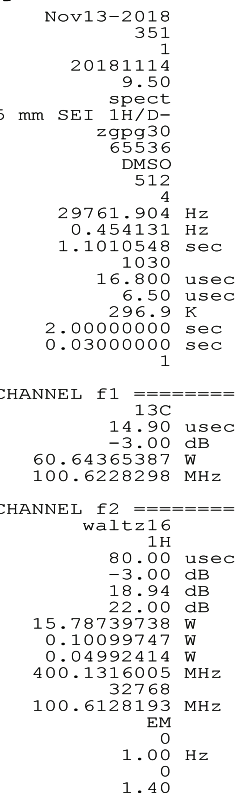

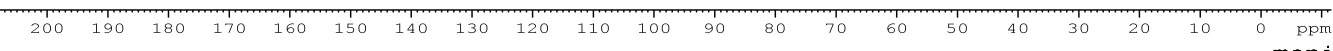

manishkumarmanu1986@gmail.cor

Figure. S13 c . APT spectra of DP1.

EFO OP3
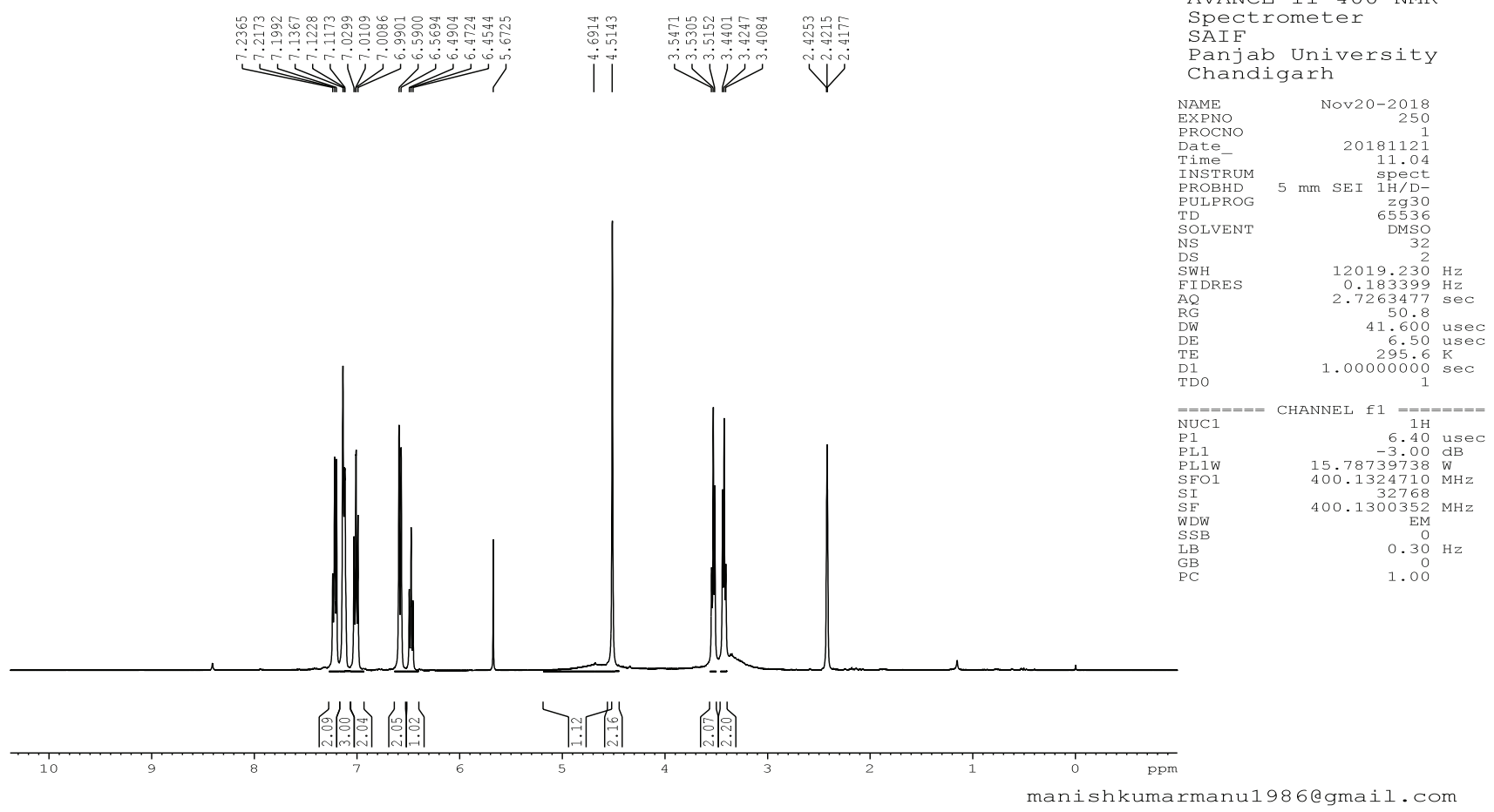

Figure. S14 a.${ }^{1} \mathrm{H}$ NMR spectra of DP3. 
EFO OP 3

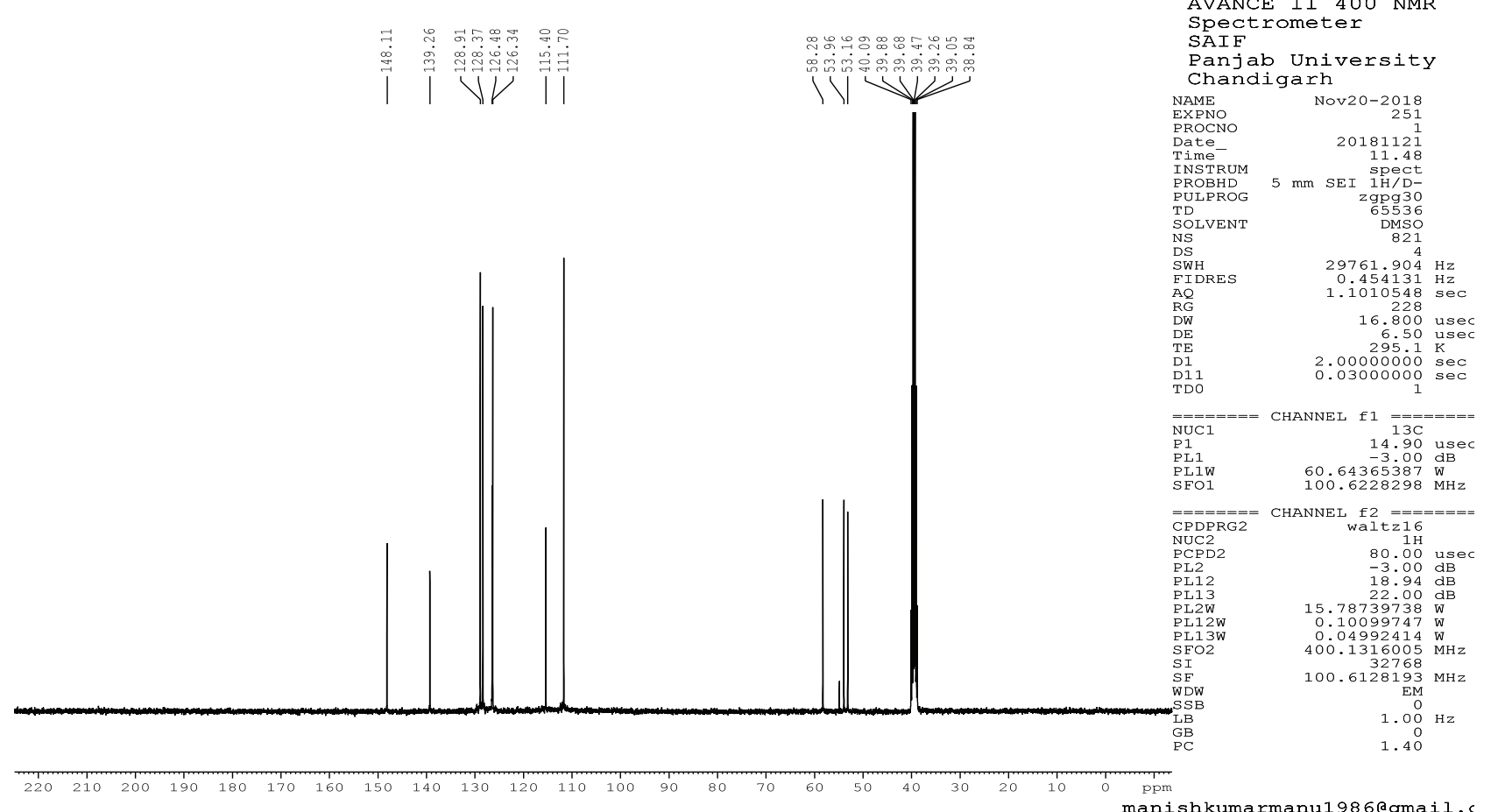

Figure. S14 b. ${ }^{13} \mathrm{C}$ NMR spectra of DP3.

EFO OP3

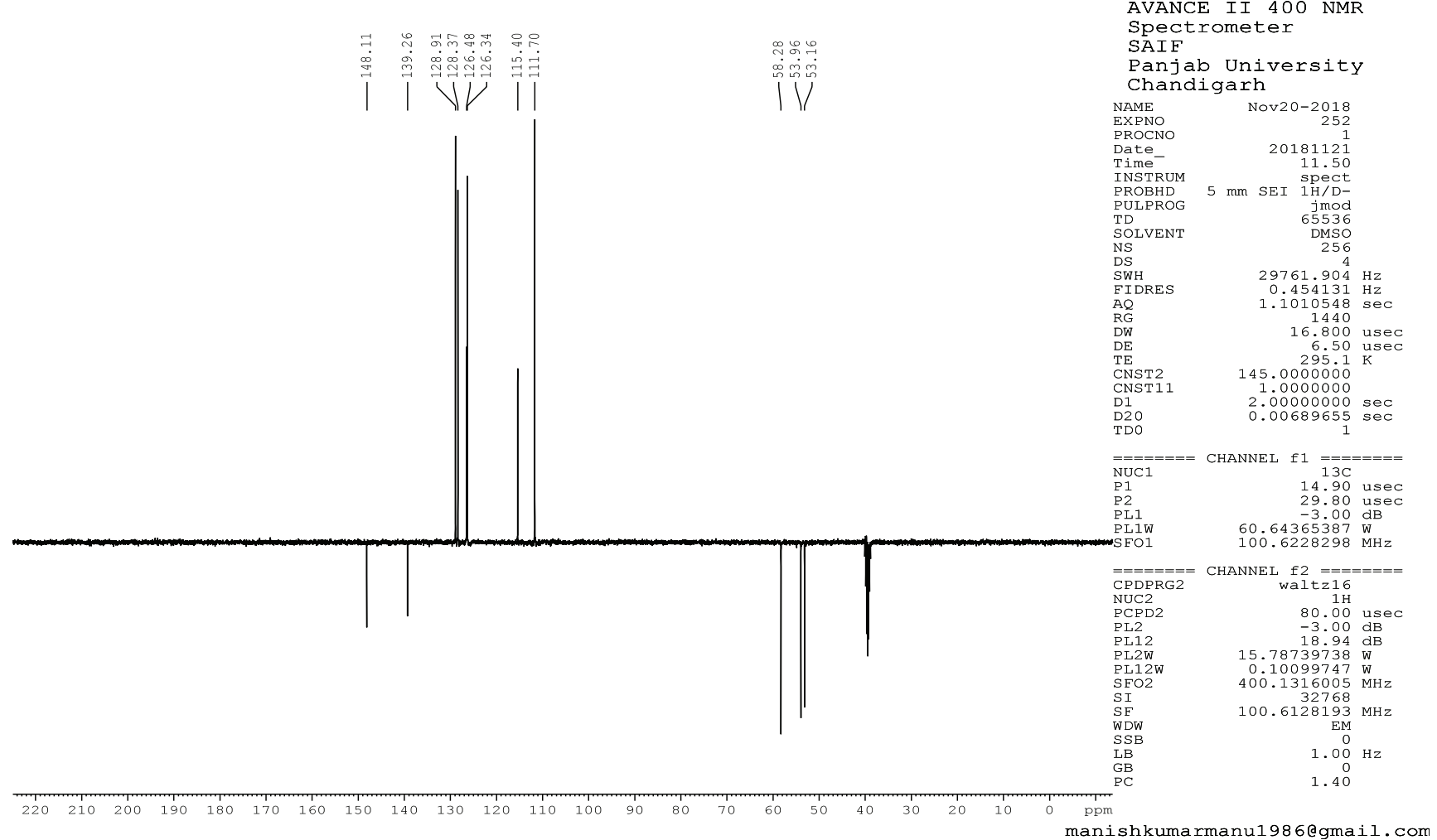

Figure. S14 c. APT spectra of DP3. 


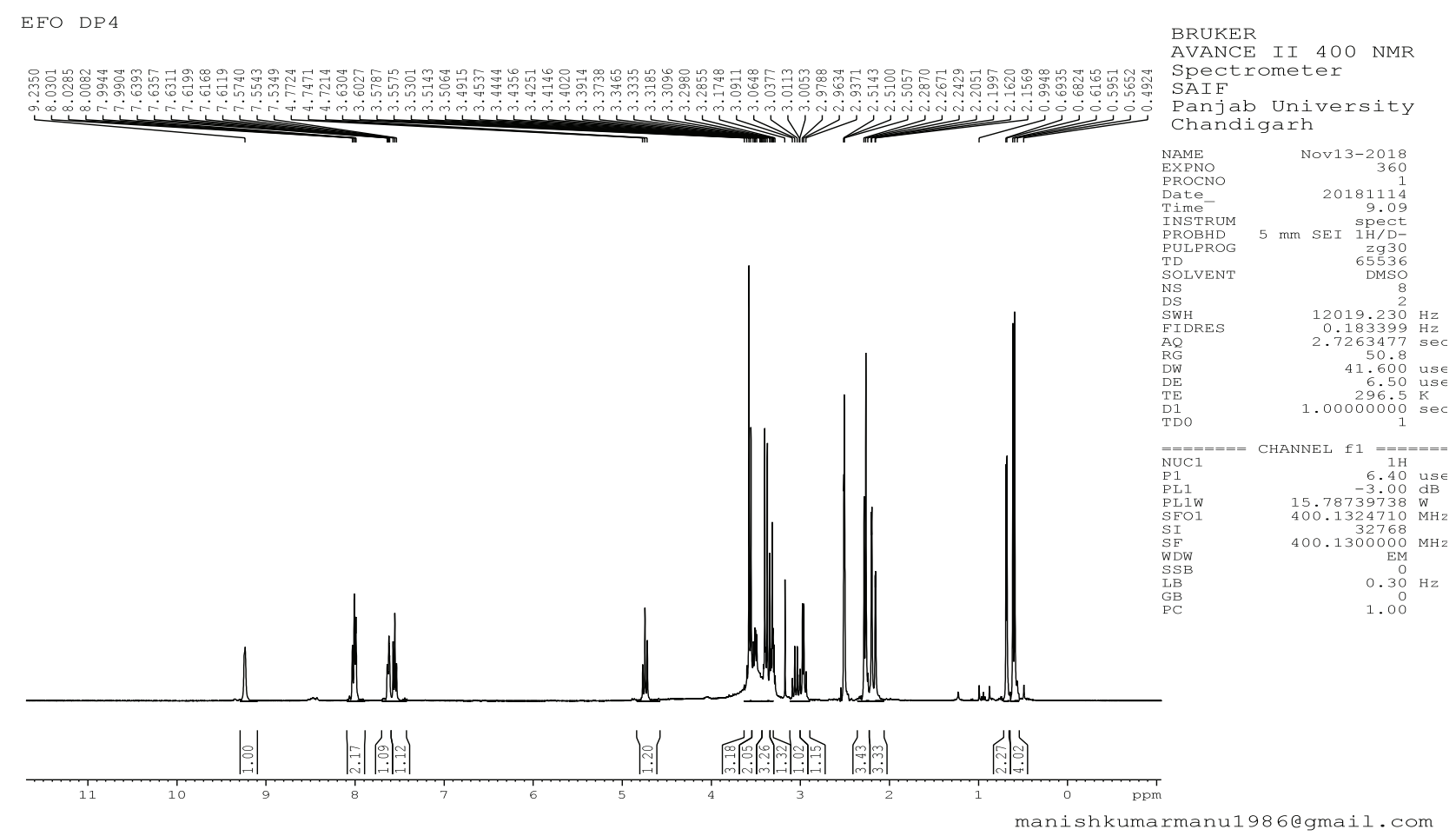

Figure. S15 a. ${ }^{1}$ H NMR spectra of DP4.

EFO DP4

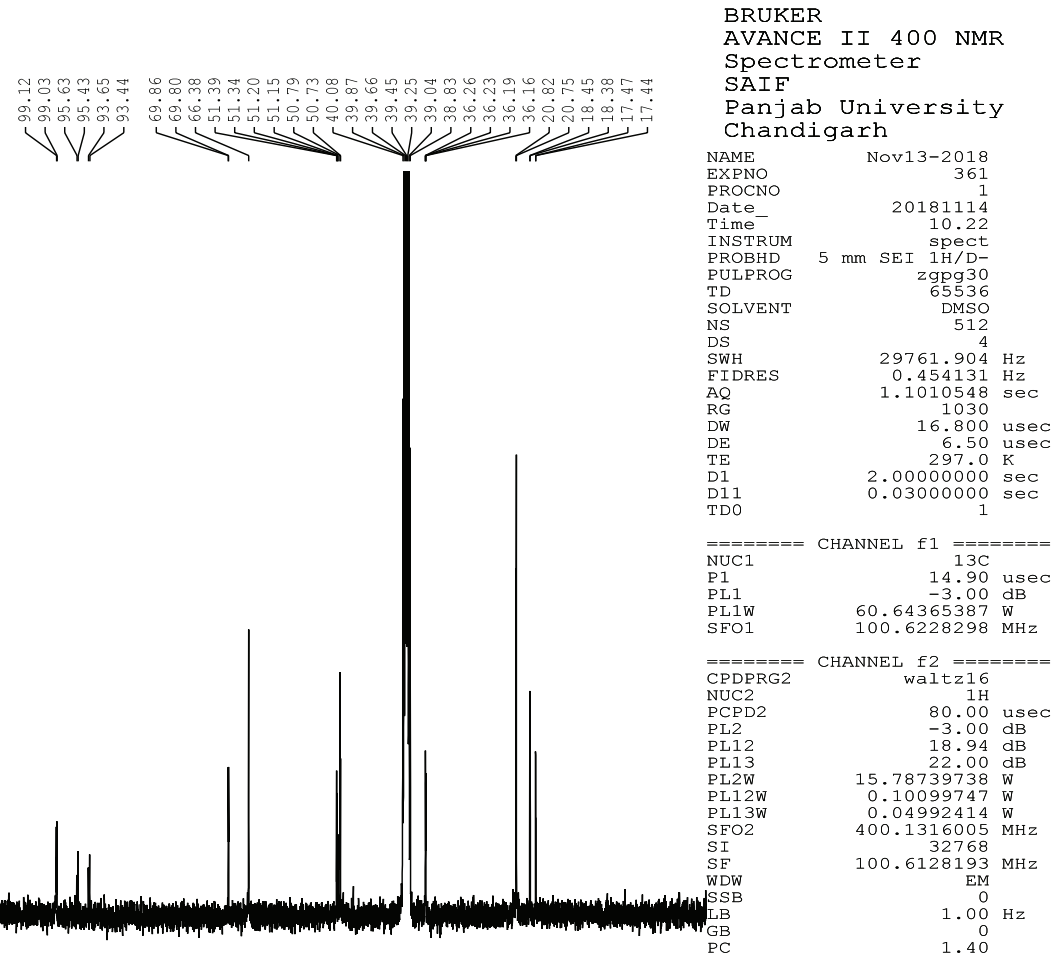

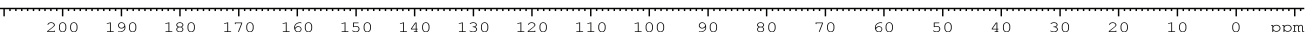

Figure. S15 b . ${ }^{13} \mathrm{C}$ NMR spectra of DP4. 
EFO DP4

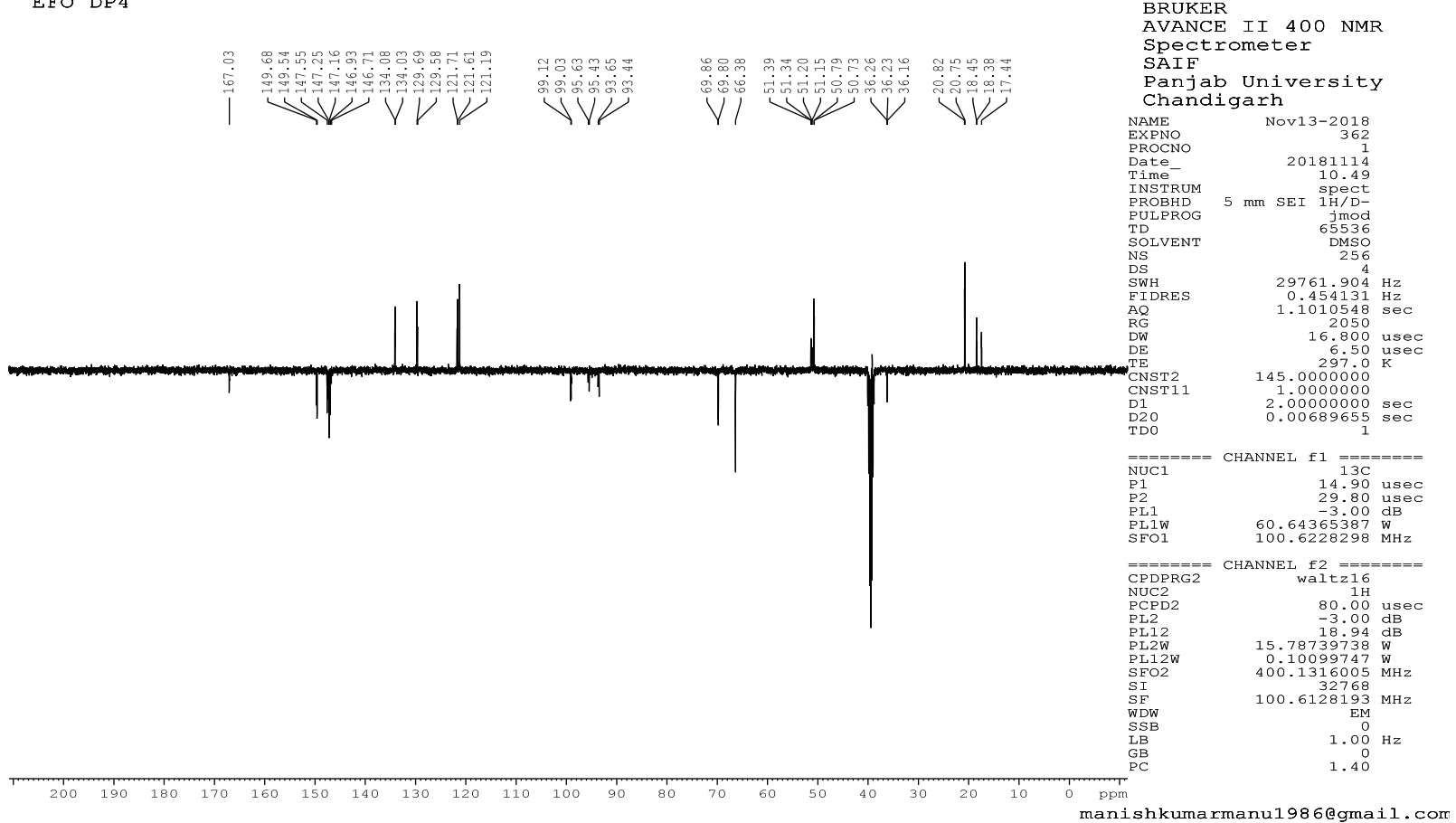

Figure. S15 c. APT spectra of DP4.

Table S1. NMR assignments of EFO.

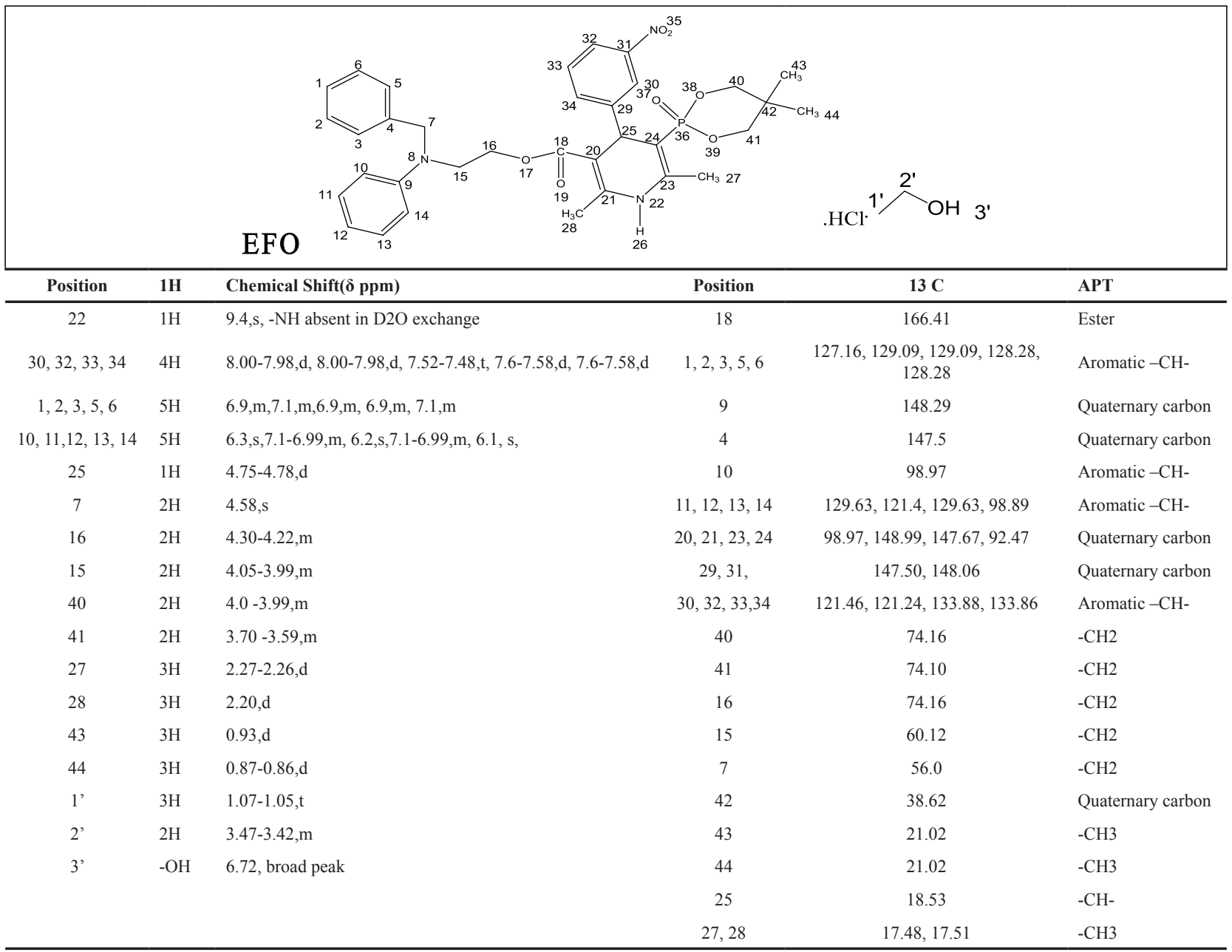


Table S2. NMR assignments of DP1.

\begin{tabular}{|c|c|c|c|c|c|}
\hline Position & $1 \mathrm{H}$ & Chemical Shift( $\delta$ ppm) & Position & $13 \mathrm{C}$ & APT \\
\hline 22 & $1 \mathrm{H}$ & $9.16, \mathrm{~s}$ & 18 & 166.64 & Ester group \\
\hline $1,2,3,5,6$ & $5 \mathrm{H}$ & $7.16-7.13, \mathrm{t}, 7.29-7.25, \mathrm{t}, 6.60-6.56, \mathrm{t}, 7.16-7.14, \mathrm{t}, 7.29-7.25, \mathrm{t}$ & $1,2,3,5,6$ & $129.56,129.56,126.59,126.59,129.56$ & Aromatic $-\mathrm{CH}$ \\
\hline $\begin{array}{c}10,11,12 \\
13,14\end{array}$ & $5 \mathrm{H}$ & $7.10-7.07, \mathrm{t}, 7.22-7.18, \mathrm{t}, 6.66-6.63, \mathrm{~m}, 7.10-7.07, \mathrm{t}, 6.66-6.63, \mathrm{~m}$ & 4 & 138.86 & Quaternary carbon \\
\hline $30,32,33,34$ & $1 \mathrm{H}$ & $8.04-8.00, \mathrm{t}, 8.04-8.00, \mathrm{t}, 7.53-7.49, \mathrm{t}, 7.62-7.64, \mathrm{~d}$ & 9 & 149.47 & Quaternary carbon \\
\hline 25 & $1 \mathrm{H}$ & $4.81, \mathrm{t}$ & $10,11,12,13,14$ & $115.99,129.68,121.20,129.68,115.99$ & Aromatic -CH \\
\hline 7 & $2 \mathrm{H}$ & $4.52-4.49, \mathrm{~d}$ & $20,21,23,24$ & $98.98,149.63,147.59,93.69$ & Quaternary carbon \\
\hline 16 & $2 \mathrm{H}$ & $4.205-4.17, \mathrm{~m}$ & 29,31 & $146.81,147.67$ & Quaternary carbon \\
\hline 15 & $2 \mathrm{H}$ & $3.66-3.50, \mathrm{~m}$ & $30,32,33,34$ & $121.68,121.68,129.68,134.15$ & Aromatic - $\mathrm{CH}-$ \\
\hline 40 & $2 \mathrm{H}$ & $3.55-3.51, \mathrm{~m}, 3.34-3.31, \mathrm{~m}$ & 7 & 49.40 & $-\mathrm{CH} 2$ \\
\hline 41 & $2 \mathrm{H}$ & $3.07-2.97, \mathrm{~m}$ & 40 & 69.89 & $-\mathrm{CH} 2$ \\
\hline 27 & $3 \mathrm{H}$ & $2.17, \mathrm{~m}$ & 41 & 66.41 & $-\mathrm{CH} 2$ \\
\hline 28 & $3 \mathrm{H}$ & $2.21, \mathrm{~m}$ & 16 & 60.52 & $-\mathrm{CH} 2$ \\
\hline 43 & $3 \mathrm{H}$ & $0.70, \mathrm{~d}$ & 15 & 53.79 & $-\mathrm{CH} 2$ \\
\hline 44 & $3 \mathrm{H}$ & $0.69, \mathrm{~d}$ & 46 & 51.35 & $-\mathrm{CH} 3$ \\
\hline 45 & $1 \mathrm{H}$ & $3.43-3.42, \mathrm{~m}$ & 42 & 36.17 & Quaternary carbon \\
\hline \multirow[t]{3}{*}{46} & $3 \mathrm{H}$ & $3.42-3.35, \mathrm{~m}$ & 25 & 18.54 & $-\mathrm{CH}-$ \\
\hline & & & 43 & 17.47 & $-\mathrm{CH} 3$ \\
\hline & & & 44 & 18.49 & $-\mathrm{CH} 3$ \\
\hline
\end{tabular}

Table S3. NMR assignments of DP3.

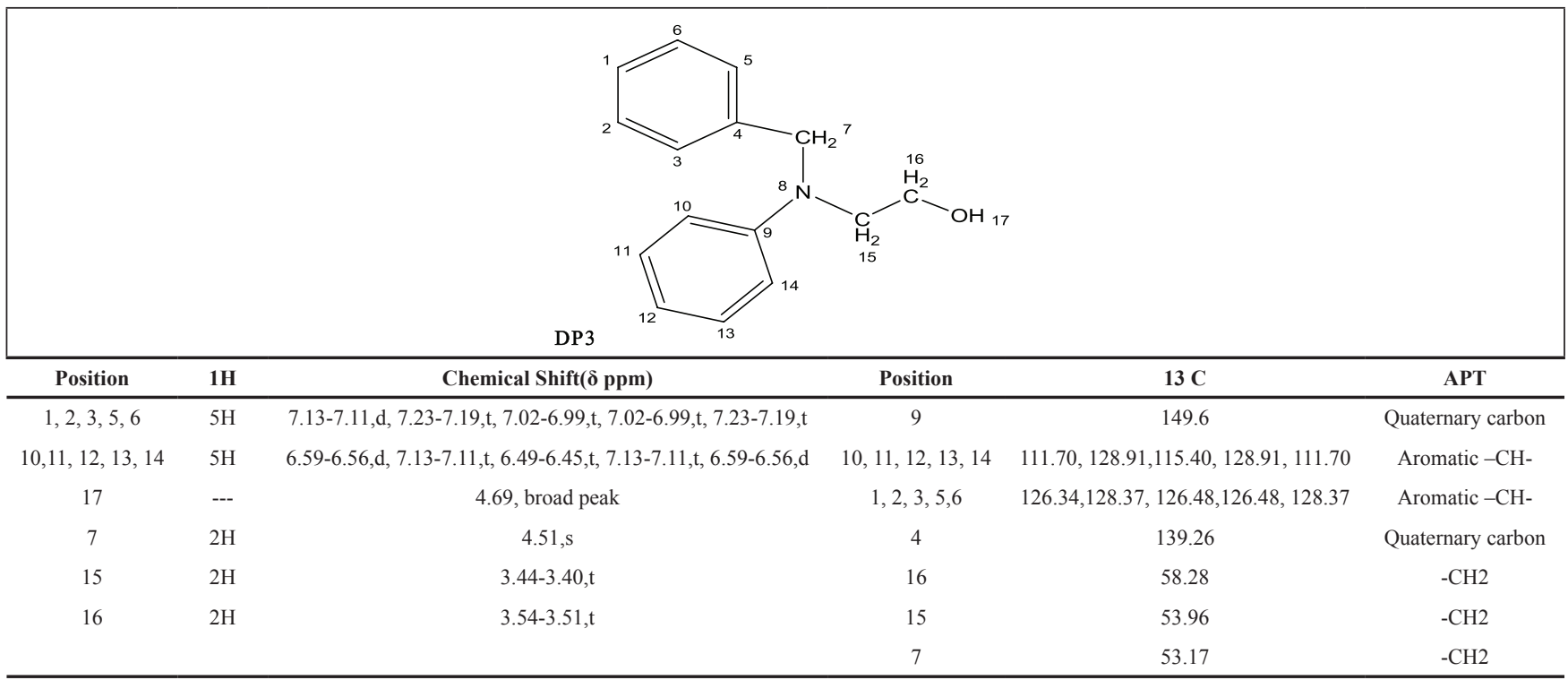


Table S4. NMR assignments of DP4.

\begin{tabular}{|c|c|c|c|c|c|}
\hline & & \begin{tabular}{ll|}
$\prod_{2}$ & $\|_{4}$ \\
4 \\
$\mathrm{H}_{3} \mathrm{C}$ \\
13
\end{tabular} & $\mathrm{NO}^{20}$ & $\underbrace{30}_{26}$ & \\
\hline Position & $1 \mathrm{H}$ & Chemical Shift $(\delta$ ppm $)$ & Position & $13 \mathrm{C}$ & APT \\
\hline 7 & $1 \mathrm{H}$ & 9.2 & 3 & 167.03 & Ester \\
\hline & & & $5,6,8,9$ & $99.12,149.58,147.25,95.63$ & Quaternary carbon \\
\hline $15,17,18,19$ & $4 \mathrm{H}$ & $\begin{array}{c}8.0-7.99, \mathrm{t}, \\
8.0-7.99, \mathrm{t}, 7.57-7.53, \mathrm{t}, 7.63-7.61, \mathrm{t}\end{array}$ & 14,16 & $146.71,149.54$ & Quaternary carbon \\
\hline 10 & $1 \mathrm{H}$ & $4.77-4.72, \mathrm{t}$ & $15,17,18,19$ & $121.71,121.19,129.6,135.08$ & Aromatic $-\mathrm{CH}-$ \\
\hline 1 & $3 \mathrm{H}$ & $3.63-3.51, \mathrm{t}$ & 24 & 69.86 & $-\mathrm{CH} 2$ \\
\hline 24 & $2 \mathrm{H}$ & $3.50-3.44, \mathrm{~m}$ & 25 & 66.38 & $-\mathrm{CH} 2$ \\
\hline 30 & $3 \mathrm{H}$ & $3.45-3.35, \mathrm{~m}$ & 1 & 51.15 & $-\mathrm{CH} 3$ \\
\hline 29 & $\begin{array}{l}-\mathrm{OH} \text {, absent in } \mathrm{D} 2 \mathrm{O} \\
\text { exchange }\end{array}$ & $3.33-3.28, \mathrm{~m}$ & 30 & 51.39 & $-\mathrm{CH} 3$ \\
\hline 25 & $2 \mathrm{H}$ & $3.0-2.9, \mathrm{~m}$ & 26 & 36.16 & Quaternary carbon \\
\hline 12 & $3 \mathrm{H}$ & $2.28-2.14, \mathrm{~d}$ & 27 & 20.75 & $-\mathrm{CH} 3$ \\
\hline 13 & $3 \mathrm{H}$ & $2.20-2.15, \mathrm{~d}$ & 28 & 20.82 & $-\mathrm{CH} 3$ \\
\hline 27 & $3 \mathrm{H}$ & $0.69-0.68, \mathrm{~d}$ & 10 & 18.45 & $-\mathrm{CH}-$ \\
\hline \multirow[t]{2}{*}{28} & $3 \mathrm{H}$ & $0.61-0.56, \mathrm{~d}$ & 12 & 18.38 & $-\mathrm{CH} 3$ \\
\hline & & & 13 & 17.44 & $-\mathrm{CH} 3$ \\
\hline
\end{tabular}

Table S5. IR spectral interpretation of EFO, DP1, DP3 and DP4.

\begin{tabular}{|c|c|c|c|}
\hline EFO & & DP1 & \\
\hline $\begin{array}{l}\text { Wave number } \\
\qquad(\mathrm{cm}-1)\end{array}$ & Assignments & $\begin{array}{l}\text { Wave number } \\
\qquad(\mathrm{cm}-1)\end{array}$ & Assignments \\
\hline 3435 & -NH Stretch & 3500 & $-\mathrm{OH}$ stretch \\
\hline 3185,3083 & Aromatic C-H Stretch & 3278 & -NH Stretch \\
\hline 2967,2860 & Alkyl C-H stretch & $3199,3088,3064$ & Aromatic C-H Stretch \\
\hline 1705 & Ester $\mathrm{C}=\mathrm{O}$ stretch & 2872 & Alkyl C-H stretch \\
\hline 1526,1494 & Aromatic nitro stretch & 2444 & Broad peak $\mathrm{O}=\mathrm{P}-\mathrm{OH}$ Stretch \\
\hline 1348,1248 & C-N stretch & 1705 & Ester \\
\hline \multirow[t]{3}{*}{1102} & $\mathrm{P}=\mathrm{O}$ stretch & 1598,1574 & $\mathrm{O}=\mathrm{P}-\mathrm{OH}$ Stretch \\
\hline & & 1528,1494 & Aromatic nitro stretch \\
\hline & & 1348,1179 & C-N stretch \\
\hline & DP4 & & DP3 \\
\hline $\begin{array}{l}\text { Wave number } \\
\qquad(\mathrm{cm}-1)\end{array}$ & Assignments & $\begin{array}{l}\text { Wave number } \\
\qquad(\mathrm{cm}-1)\end{array}$ & Assignments \\
\hline 3500 and 3400 & Broad peak covering $-\mathrm{OH}$ and $-\mathrm{NH}$ group & 3304 & Broad peak $-\mathrm{OH}$ \\
\hline 3192,3166 & Aromatic C-H stretch & 3000 & Aromatic C-H stretch \\
\hline $2951,2873,2848$ & Alkyl C-H stretch & 2970 & Alkyl stretch \\
\hline 2444 & $\mathrm{O}=\mathrm{P}-\mathrm{OH}$ Stretch & 1591,1432 & Aromatic $\mathrm{C}=\mathrm{C}$ Stretch \\
\hline 1740 & Ester $\mathrm{C}=\mathrm{O}$ stretch & & \\
\hline 1644,1628 & $\mathrm{O}=\mathrm{P}-\mathrm{OH}$ Stretch & 850 & Out of plane bending \\
\hline 1530,1497 & Aromatic nitro stretch & 640 & Out of plane bending \\
\hline 1348,1280 & C-N Stretch & & \\
\hline 1053 & $\mathrm{P}=\mathrm{O}$ stretch & & \\
\hline
\end{tabular}

